\title{
Backward orbits and petals of semigroups of holomorphic self-maps of the unit disc
}

\author{
Filippo Bracci $^{1}$ (D) - Manuel D. Contreras ${ }^{2}$ - Santiago Díaz-Madrigal ${ }^{2}$. \\ Hervé Gaussier ${ }^{3}$
}

Received: 14 May 2018 / Accepted: 28 July 2018 / Published online: 6 August 2018

(c) Fondazione Annali di Matematica Pura ed Applicata and Springer-Verlag GmbH Germany, part of Springer Nature 2018

\begin{abstract}
We study the backward invariant set of one-parameter semigroups of holomorphic self-maps of the unit disc. Such a set is foliated in maximal invariant curves, and its open connected components are petals, which are, in fact, images of Poggi-Corradini's type pre-models. Hyperbolic petals are in one-to-one correspondence with repelling fixed points, while only parabolic semigroups can have parabolic petals. Petals have locally connected boundaries, and except a very particular case, they are indeed Jordan domains. The boundary of a petal contains the Denjoy-Wolff point, and except such a fixed point, the closure of a petal contains either no other boundary fixed points or a unique repelling fixed point. We also describe petals in terms of geometric and analytic behavior of Koenigs functions using divergence rate and universality of models. Moreover, we construct a semigroup having a repelling fixed point in such a way that the intertwining map of the pre-model is not regular.
\end{abstract}

Keywords Semigroups of holomorphic functions $\cdot$ Backward orbits $\cdot$ Petals $\cdot$ Koenigs function · Holomorphic models

Mathematics Subject Classification Primary 37C10 - 30C35; Secondary 30D05 - 30C80 . 37F99 - 37C25

Filippo Bracci: Partially supported by GNSAGA of INdAM and by the MIUR Excellence Department Project awarded to the Department of Mathematics, University of Rome Tor Vergata, CUP E83C18000100006.

Manuel D. Contreras, Santiago Díaz-Madrigal: Partially supported by the Ministerio de Economía y Competitividad and the European Union (FEDER) MTM2015-63699-P and by La Consejería de Educación y Ciencia de la Junta de Andalucía.

$凶 \quad$ Filippo Bracci

fbracci@mat.uniroma2.it

Extended author information available on the last page of the article 


\section{Contents}

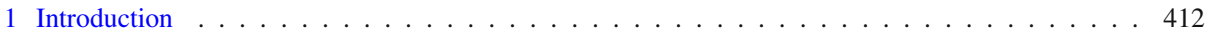

2 Preliminaries ............................ 413

3 Backward orbits and pre-models . . . . . . . . . . . . . . . . . . . . 415

4 Petals ............................... 418

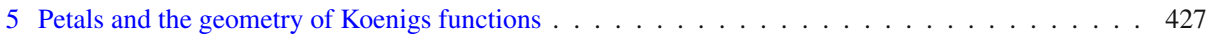

6 Analytic properties of Koenigs functions at boundary fixed points . . . . . . . . . . . . . . 431

7 Examples . . . . . . . . . . . . . . . . . . . . . . . 434

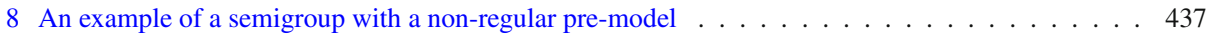

References . . . . . . . . . . . . . . . . . . . . . . . . . . . . . . . . . . . 440

\section{Introduction}

One-parameter continuous semigroups of holomorphic self-maps of $\mathbb{D}$ —for short, holomorphic semigroups in $\mathbb{D}$ — have been widely studied; see, for example, [1,4,27,29]. In this paper, we study the behavior of semigroups at the boundary from a dynamical point of view, with special attention to boundary regular (in particular repelling) fixed points, a subject that has been addressed in a number of recent papers [9-11,16-18].

Given a semigroup $\left(\phi_{t}\right)$ in $\mathbb{D}$ and a point $z \in \mathbb{D}$, one can follow the "backward" trajectory up to a boundary point. The union of the backward trajectory and the forward trajectory of $z$ is a maximal invariant curve for the semigroup. In case the backward trajectory is defined for all negative times, it is called a backward orbit.

Backward orbits for discrete holomorphic iteration in the unit disc have been introduced in [21], where Poggi-Corradini proved that for every repelling fixed point of a holomorphic self-map of $\mathbb{D}$, there exists a backward orbit with bounded hyperbolic step. Using such an orbit as basis for a suitable rescaling, Poggi-Corradini showed the existence of pre-models. Abstract backward orbits for discrete iteration have been studied by the first named author in [5], with the aim of proving a conjecture of Cowen [13] about common boundary fixed points of commuting holomorphic maps. Later, Poggi-Corradini [22,23] gave a systematic treatment of the subject, and, recently, Arosio [2] focused on backward orbits and pre-models with a categorial point of view which holds also in higher dimension. The previously cited results can be clearly adapted to holomorphic semigroups in $\mathbb{D}$, and we collect them in Sect. 3 .

We point out that, in Poggi-Corradini [21], the author proved that the intertwining map of a pre-model at a repelling fixed point is always semi-conformal, and he gave a (rather complicated) example of a holomorphic self-map of $\mathbb{D}$ for which the intertwining map of the pre-model is not regular. In Sect. 8, using suitable localization of the hyperbolic distance, we construct a holomorphic semigroup of $\mathbb{D}$ for which the intertwining map of the pre-model at a repelling fixed point is not regular.

The core and the main novelties of the paper are contained in Sect. 4. There we start considering the backward invariant set $\mathscr{W}$ of a holomorphic semigroup of $\mathbb{D}$, namely the union of those points for which the backward trajectories are defined for every negative times. This set is foliated in real analytic curves (which we call maximal invariant curves) which are orbits along which the Cauchy problem for the infinitesimal generator of the semigroup has a maximal solution defined for every real time. The interior of $\mathscr{W}$, the boundary of $\mathscr{W}$ and its complement are all completely $\left(\phi_{t}\right)$-invariant. The connected components of the interior of $\mathscr{W}$ are called petals. All petals contain the Denjoy-Wolff point of $\left(\phi_{t}\right)$ in their closure. We call a petal parabolic if it contains only a fixed point of the semigroup - that is, the Denjoy- 
Wolff point-in its closure, hyperbolic otherwise. The main new results in the paper can be summarized in the following theorem:

Theorem 1.1 Let $\left(\phi_{t}\right)$ be a holomorphic semigroup in $\mathbb{D}$, not an elliptic group, with DenjoyWolff point $\tau \in \overline{\mathbb{D}}$. Let $\Delta$ be a petal of $\left(\phi_{t}\right)$. Then,

(1) $\Delta$ is simply connected and $\tau \in \partial \Delta$,

(2) $\phi_{t}(\Delta)=\Delta$ and $\left(\left.\phi_{t}\right|_{\Delta}\right)$ is a continuous one-parameter group of $\Delta$.

(3) $\partial \Delta$ is locally connected and, except a very particular case, $\Delta$ is a Jordan domain. There are, in fact, only five possible types of petals (see Proposition 4.15 for a precise description).

(4) $\bar{\Delta} \backslash \tau$ contains at most one boundary fixed point $\sigma \in \partial \mathbb{D}$ of $\left(\phi_{t}\right)$. If this is the case, that is, $\Delta$ is hyperbolic, then $\sigma$ is a repelling fixed point of $\left(\phi_{t}\right)$.

(5) If $\Delta$ is parabolic, then $\left(\phi_{t}\right)$ is a parabolic semigroup.

(6) There is a one-to-one correspondence between repelling fixed points of $\left(\phi_{t}\right)$ and hyperbolic petals.

(7) For every $z \in \Delta$, the curve $[0,+\infty) \mapsto \phi_{-t}(z)$ is a regular backward orbit for the semigroup (see Definition 3.1).

(8) If $\Delta$ is hyperbolic and $\sigma \in \partial \Delta \backslash\{\tau\}$ is the (unique) repelling fixed point of $\left(\phi_{t}\right)$ contained in $\bar{\Delta}$, then, for every $z \in \Delta$ it holds $\lim _{t \rightarrow-\infty} \frac{1}{t} \log \left(1-\bar{\sigma} \phi_{t}(z)\right)=-\lambda$, where $\lambda \in$ $(-\infty, 0)$ is the repelling spectral value of $\left(\phi_{t}\right)$ at $\sigma$ (see Definition 2.1).

As a corollary of the previous result, we prove that if $\sigma \in \partial \mathbb{D}$ is a boundary fixed point which is not regular (also called super-repelling fixed point), then there exists at most one backward orbit of $\left(\phi_{t}\right)$ landing at $\sigma$ (see Proposition 4.21).

In case of an elliptic and starlike holomorphic self-map of $\mathbb{D}$, Poggi-Corradini proved that every repelling fixed point corresponds to a sector in the image domain of the Koenigs function with amplitude related to the boundary dilation coefficient at the fixed point. This result, and its converse, for every case for semigroups was proved with a direct, lengthy and rather complicated argument (which needs to consider the type of semigroup case by case) by the second and third author in [9].

The next aim of this paper is to give a simple comprehensive proof of those results (see Theorems 5.6 and 5.7). The idea is that, since on each hyperbolic petal the semigroup acts as a hyperbolic group, the restriction of the Koenigs function on the petal is a holomorphic model in the sense of [3]. Then, the rate of divergence-which is a measure of how fast an orbit escapes to the Denjoy-Wolff point—of the semigroup on the petal has to be the same for the model via the Koenigs function. The divergence rate "upstairs" is essentially given by the repelling spectral value of the semigroup at the corresponding repelling fixed point, and hence, "downstairs"; this forces the shape of the image of the Koenigs function (see Sect. 5 for details).

In Sect. 6, we use the previously proved results to characterize the analytic behavior of the Koenigs function of the semigroup at boundary points.

Finally, in Sect. 7, we provide several examples and in Sect. 8 we construct an example of a holomorphic semigroup having a pre-model at a repelling fixed point whose intertwining map is not regular at such a point.

We thank the referee for useful comments which improved the original manuscript.

\section{Preliminaries}

For all the statements without references, we refer the reader to, for example, [1,14] or [26]. 
A continuous one-parameter semigroup $\left(\phi_{t}\right)$ of holomorphic self-maps of $\mathbb{D}$-a holomorphic semigroup of $\mathbb{D}$ for short—is a continuous homomorphism $t \mapsto \phi_{t}$ from the additive semigroup $\left(\mathbb{R}_{\geq 0},+\right)$ of nonnegative real numbers to the semigroup ( $\mathrm{Hol}(\mathbb{D}, \mathbb{D})$, o) of holomorphic self-maps of $\mathbb{D}$ with respect to composition, endowed with the topology of uniform convergence on compacta.

If $\phi_{t_{0}}$ is an automorphism of $\mathbb{D}$ for some $t_{0}>0$, then $\phi_{t}$ is an automorphism of $\mathbb{D}$ for all $t \geq 0$ and the semigroup can be extended to a group.

If $\left(\phi_{t}\right)$ is not a group of hyperbolic rotations, namely it does not contain elliptic automorphisms of $\mathbb{D}$, then there exists a unique point $\tau \in \overline{\mathbb{D}}$ such that $\phi_{t}$ converges uniformly on compacta to the constant map $z \mapsto \tau$. Such a point $\tau$ is called the Denjoy-Wolff point of $\left(\phi_{t}\right)$.

The semigroup is called elliptic if $\tau \in \mathbb{D}$. In such a case, $\phi_{t}(\tau)=e^{-\lambda t}$ with $\lambda \in \mathbb{C}$, $\operatorname{Re} \lambda>0$.

If $\tau \in \partial \mathbb{D}$, then the non-tangential limit $\angle \lim _{z \rightarrow \tau} \phi_{t}(z)=\tau$ for all $t \geq 0$ and $\angle \lim _{z \rightarrow \tau} \phi_{t}^{\prime}(z)=e^{-\lambda t}$ for some $\lambda \geq 0$. In case $\lambda>0$ the semigroup is called hyperbolic, while, if $\lambda=0$ the semigroup is parabolic. The number $\lambda$ is called the dilation (or the spectral value) of $\left(\phi_{t}\right)$.

Parabolic semigroups can be divided in two sub-types: a parabolic holomorphic semigroup in $\mathbb{D}$ is of positive hyperbolic step if $\lim _{t \rightarrow+\infty} \omega\left(\phi_{t+1}(0), \phi_{t}(0)\right)>0$ (here $\omega(z, w)$ is the hyperbolic distance in $\mathbb{D}$ between $z \in \mathbb{D}$ and $w \in \mathbb{D}$ ). Otherwise, it is called of zero hyperbolic step.

Definition 2.1 A point $\sigma \in \partial \mathbb{D}$ is a boundary fixed point of $\left(\phi_{t}\right)$ if $\angle \lim _{z \rightarrow \sigma} \phi_{t}(z)=\sigma$ for all $t \geq 0$.

Moreover, a boundary fixed point $\sigma \in \partial \mathbb{D}$ is called a boundary regular fixed point if $\angle \lim _{z \rightarrow \sigma} \phi_{t}^{\prime}(z)=e^{-\mu t}$ for some $\mu \in \mathbb{R}$ and for all $t \geq 0$. If $\mu<0$, the point $\sigma$ is a repelling fixed point of $\left(\phi_{t}\right)$. In this case, the number $\mu$ is called the repelling spectral value of $\left(\phi_{t}\right)$ at $\sigma$.

A boundary fixed point which is not regular is called a super-repelling fixed point.

We point out for the reader's convenience that the previous definition is not the standard definition of a boundary regular fixed point for a holomorphic self-map of $\mathbb{D}$, but it is equivalent to that (see [1, Prop. 1.2.8]), and it is enough for our aims. We also note that, apart from the trivial semigroup, the only boundary regular fixed points of a semigroup are the Denjoy-Wolff point (provided the semigroup is not elliptic) and repelling fixed points.

It is known (see, [10, Theorem 1], [11, Theorem 2], [28, pag. 255], [15]) that a point $\sigma \in \partial \mathbb{D}$ is a boundary (regular) fixed point of $\phi_{t_{0}}$ for some $t_{0}>0$ if and only if it is a boundary (regular) fixed point of $\phi_{t}$ for all $t \geq 0$.

By Berkson-Porta's theorem [4, Theorem (1.1)], if $\left(\phi_{t}\right)$ is a holomorphic semigroup in $\mathbb{D}$, then $t \mapsto \phi_{t}(z)$ is real analytic and there exists a unique holomorphic vector field $G: \mathbb{D} \rightarrow \mathbb{C}$ such that $\frac{\partial \phi_{t}(z)}{\partial t}=G\left(\phi_{t}(z)\right)$ for all $z \in \mathbb{D}$ and all $t \geq 0$. This vector field $G$ - the infinitesimal generator of $\left(\phi_{t}\right)$-is semicomplete in the sense that the associated Cauchy problem

$$
\left\{\begin{array}{l}
\frac{\mathrm{d} x(t)}{\mathrm{d} t}=G(x(t)), \\
x(0)=z,
\end{array}\right.
$$

has a solution $x^{z}:[0,+\infty) \rightarrow \mathbb{D}$ for every $z \in \mathbb{D}$. Conversely, any semicomplete holomorphic vector field in $\mathbb{D}$ generates a continuous one-parameter semigroup of holomorphic self-maps of $\mathbb{D}$.

Another key notion associated with semigroups is that of holomorphic model. 
Definition 2.2 Let $\left(\phi_{t}\right)$ be a semigroup of holomorphic self-maps of $\mathbb{D}$. A (holomorphic) model for $\left(\phi_{t}\right)$ is a triple $\left(\Omega, h, \Phi_{t}\right)$ such that $\Omega$ is an open subset of $\mathbb{C}, \Phi_{t}$ is a group of (holomorphic) automorphisms of $\Omega$ and $h: \mathbb{D} \rightarrow h(\mathbb{D}) \subset \Omega$ is univalent on the image, $h \circ \phi_{t}=\Phi_{t} \circ h$ and

$$
\cup_{t \geq 0} \Phi_{t}^{-1}(h(\mathbb{D}))=\Omega .
$$

The previous notion of holomorphic model was introduced in [3], where it was proved that every semigroup of holomorphic self-maps of any complex manifold admits holomorphic models and they are unique up to holomorphic equivalence of models. Moreover, a model is "universal" in the sense that every other conjugation of the semigroup to a group of automorphisms factorizes through the model (see [3, Section 6] for more details).

Notice that given a model $\left(\Omega, h, \Phi_{t}\right)$ for a semigroup $\left(\phi_{t}\right)$ of holomorphic self-maps of $\mathbb{D}$, then $\left(\phi_{t}\right)$ is a group if and only if $h(\mathbb{D})=\Omega$.

In what follows, we denote by $\mathbb{H}:=\{\zeta \in \mathbb{C}: \operatorname{Re} \zeta>0\}, \mathbb{H}^{-}:=\{\zeta \in \mathbb{C}: \operatorname{Re} \zeta<0\}$ and, given $\rho>0, \mathbb{S}_{\rho}:=\{\zeta \in \mathbb{C}: 0<\operatorname{Re} \zeta<\rho\}$. We simply write $\mathbb{S}:=\mathbb{S}_{1}$. The following result sums up the results in $[3,12]$; see also [1].

Theorem 2.3 Let $\left(\phi_{t}\right)$ be a semigroup in $\mathbb{D}$. Then,

(1) $\left(\phi_{t}\right)$ is the trivial semigroup if and only if $\left(\phi_{t}\right)$ has a holomorphic model $\left(\mathbb{D}, i d_{\mathbb{D}}, z \mapsto z\right)$.

(2) $\left(\phi_{t}\right)$ is a group of elliptic automorphisms with spectral value $i \theta$, for $\theta \in \mathbb{R} \backslash\{0\}$, if and only if $\left(\phi_{t}\right)$ has a holomorphic model $\left(\mathbb{D}, h, z \mapsto e^{-i \theta t} z\right)$.

(3) ( $\left.\phi_{t}\right)$ is elliptic, not a group, with spectral value $\lambda$, for $\lambda \in \mathbb{C}$ with $\operatorname{Re} \lambda>0$, if and only if $\left(\phi_{t}\right)$ has a holomorphic model $\left(\mathbb{C}, h, z \mapsto e^{-\lambda t} z\right)$.

(4) $\left(\phi_{t}\right)$ is hyperbolic with spectral value $\lambda>0$ if and only if it has a holomorphic model $\left(\mathbb{S}_{\frac{\pi}{2}}, h, z \mapsto z+i t\right)$.

(5) $\left(\phi_{t}\right)$ is parabolic of positive hyperbolic step if and only if it has a holomorphic model either of the form $(\mathbb{H}, h, z \mapsto z+i t)$ or of the form $\left(\mathbb{H}^{-}, h, z \mapsto z+i t\right)$.

(6) $\left(\phi_{t}\right)$ is parabolic of zero hyperbolic step if and only if it has a holomorphic model $(\mathbb{C}, h, z \mapsto z+i t)$.

The holomorphic models defined in the previous theorem are called canonical. The function $h$ in the canonical model of $\left(\phi_{t}\right)$ is called the Koenigs function of the semigroup.

Finally, in this paper we will make use of Carathéodory's prime ends theory. We refer the reader to Pommerenke's books [24,25] and Collingwood and Lohwater's book [8] for all nonproven statements about it.

\section{Backward orbits and pre-models}

For $z, w \in \mathbb{D}$, we let $\omega(z, w)$ be the hyperbolic distance in $\mathbb{D}$ of $z, w$.

Definition 3.1 Let $\left(\phi_{t}\right)$ be a semigroup in $\mathbb{D}$. A continuous curve $\gamma:[0,+\infty) \rightarrow \mathbb{D}$ is called a backward orbit if for every $t \in(0,+\infty)$ and for every $0 \leq s \leq t$,

$$
\phi_{s}(\gamma(t))=\gamma(t-s) \text {. }
$$

A backward orbit $\gamma$ is said to be a regular backward orbit if

$$
V(\gamma):=\limsup _{t \rightarrow+\infty} \omega(\gamma(t), \gamma(t+1))<+\infty .
$$

We call $V(\gamma)$ the hyperbolic step of $\gamma$. 
Remark 3.2 Let $\left(\phi_{t}\right)$ be a semigroup in $\mathbb{D}$ and let $\gamma:[0,+\infty) \rightarrow \mathbb{D}$ be a backward orbit for $\left(\phi_{t}\right)$. Let $\left(\Omega, h, \psi_{t}\right)$ be the canonical model of $\left(\phi_{t}\right)$ given by Theorem 2.3. For all $t \geq 0$, $h(\gamma(t)) \in h(\mathbb{D})$, hence, $\psi_{t}(h(\gamma(t)))=h\left(\phi_{t}(\gamma(t))\right)=h(\gamma(0))$ and

$$
h(\gamma(t))=\psi_{-t}(h(\gamma(0))), \quad \text { for all } t \geq 0 .
$$

In particular, if $\left(\phi_{t}\right)$ is elliptic, not a group, and $\lambda \in \mathbb{C}, \operatorname{Re} \lambda>0$ is its spectral value, then

$$
h(\gamma([0,+\infty)))=\left\{e^{-\lambda s} h(\gamma(0)): s \in \mathbb{R}\right\} \cap\{w \in \mathbb{C}:|w| \geq|h(\gamma(0))|\} .
$$

While, if $\left(\phi_{t}\right)$ is non-elliptic,

$$
h(\gamma([0,+\infty)))=\{w \in \mathbb{C}: \operatorname{Re} w=\operatorname{Re} h(\gamma(0))\} \cap\{w \in \mathbb{C}: \operatorname{Im} w \leq \operatorname{Im} h(\gamma(0))\} .
$$

The study of backward orbits of groups is particularly easy by direct computation and we leave the details for the reader:

Proposition 3.3 Let $\left(\phi_{t}\right)$ be a non-trivial group in $\mathbb{D}$ and let $\tau \in \overline{\mathbb{D}}$ be the Denjoy-Wolff point of $\left(\phi_{t}\right)$. Let $\gamma:[0,+\infty) \rightarrow \mathbb{D}$ be a backward orbit. Then, $\gamma$ is regular. Moreover,

(1) if $\left(\phi_{t}\right)$ is elliptic, then either $\gamma(t) \equiv \tau$ or the image of $\gamma$ is the boundary of a hyperbolic disc centered at $\tau$.

(2) If $\left(\phi_{t}\right)$ is hyperbolic and $\sigma \in \partial \mathbb{D} \backslash\{\tau\}$ is the other fixed point of $\left(\phi_{t}\right)$, then $\lim _{t \rightarrow+\infty} \gamma(t)=\sigma$ and there exists $\alpha \in(-\pi / 2, \pi / 2)$ such that $\lim _{t \rightarrow+\infty} \operatorname{Arg}(1-$ $\bar{\sigma} \gamma(t))=\alpha$.

(3) If $\left(\phi_{t}\right)$ is parabolic, then $\lim _{t \rightarrow+\infty} \gamma(t)=\tau$ and $|\tau-\gamma(t)|^{2}=R\left(1-|\gamma(t)|^{2}\right)$ for some $R>0$ and for all $t \geq 0$. In particular, $\gamma(t)$ converges to $\tau$ tangentially.

Remark 3.4 It is easy to see that if $\left(\phi_{t}\right)$ is a hyperbolic group with Denjoy-Wolff point $\tau \in \partial \mathbb{D}$ and $\sigma \in \partial \mathbb{D} \backslash\{\tau\}$ is other fixed point, then for every $\alpha \in(-\pi / 2, \pi / 2)$ there exists $z_{0} \in \mathbb{D}$ such that $[0,+\infty) \ni t \mapsto \phi_{-t}\left(z_{0}\right)$ is a regular backward orbit which converges to $\sigma$ and such that $\lim _{t \rightarrow+\infty} \operatorname{Arg}\left(1-\bar{\sigma} \phi_{-t}\left(z_{0}\right)\right)=\alpha$.

Now, we examine the case of semigroups which are not groups. We start with the following result:

Lemma 3.5 Let $\left(\phi_{t}\right)$ be a semigroup in $\mathbb{D}$ which is not a group. Let $\tau \in \overline{\mathbb{D}}$ be its Denjoy-Wolff point. Let $\gamma:[0,+\infty) \rightarrow \mathbb{D}$ be a backward orbit for $\left(\phi_{t}\right)$. Then,

- either $\tau \in \mathbb{D}$ and $\gamma(t) \equiv \tau$ for all $t \geq 0$,

- or there exists $\sigma \in \partial \mathbb{D}$ (possibly $\sigma=\tau$ ) such that $\sigma$ is a fixed point of $\left(\phi_{t}\right)$ and $\lim _{t \rightarrow+\infty} \gamma(t)=\sigma$.

Proof Let $\left(\Omega, h, \psi_{t}\right)$ be the canonical model for $\left(\phi_{t}\right)$, where $\Omega$ is either $\mathbb{C}, \mathbb{H}, \mathbb{H}^{-}$or the strip $\mathbb{S}_{\rho}$ for some $\rho>0$ and either $\psi_{t}(w)=w+i t$ or $\psi_{t}(w)=e^{-\lambda t} w$ for some $\lambda \in \mathbb{C}$ with $\operatorname{Re} \lambda>0$. Let $w_{0}:=h(\gamma(0))$. Let $t \geq 0$. By Remark 3.2, $h(\gamma(t))=\psi_{-t}\left(w_{0}\right)$ for all $t \geq 0$. In particular, by the form of $\psi_{t}$, it follows that either $\psi_{-t}\left(w_{0}\right) \equiv w_{0}=0$ (and hence $\gamma(t) \equiv \tau)$ or $\lim _{t \rightarrow+\infty} \psi_{-t}\left(w_{0}\right)=\infty$ in $\mathbb{C}_{\infty}$. Therefore, by [26, Lemma 2, p. 162], there exists $\sigma \in \partial \mathbb{D}$ such that

$$
\lim _{t \rightarrow+\infty} \gamma(t)=\lim _{t \rightarrow+\infty} h^{-1}\left(h(\gamma(t))=\lim _{t \rightarrow+\infty} h^{-1}\left(\psi_{-t}\left(w_{0}\right)\right)=\sigma .\right.
$$

Now, let $s>0$. Then

$$
\lim _{t \rightarrow+\infty} \phi_{s}(\gamma(t))=\lim _{t \rightarrow+\infty} \gamma(t-s)=\sigma .
$$


Therefore, by Lehto-Virtanen Theorem, $\angle \lim _{z \rightarrow \sigma} \phi_{s}(z)=\sigma$ for all $s \geq 0$; hence $\sigma$ is a boundary fixed point of $\left(\phi_{t}\right)$.

The proof of the next result follows quite directly from the corresponding results for the discrete case (see [22, Lemma 2.1], [5,9]).

Proposition 3.6 Let $\left(\phi_{t}\right)$ be a semigroup in $\mathbb{D}$ which is not a group. Let $\tau \in \overline{\mathbb{D}}$ be its DenjoyWolff point. Let $\gamma:[0,+\infty) \rightarrow \mathbb{D}$ be a regular backward orbit for $\left(\phi_{t}\right)$. Then,

(1) either $\tau \in \mathbb{D}$ and $\gamma(t)=\tau$ for all $t \in[0,+\infty)$,

(2) or, there exists a unique point $\sigma \in \partial \mathbb{D} \backslash\{\tau\}$ such that $\gamma(t)$ converges to $\sigma$ non-tangentially. Moreover, $\sigma$ is a repelling fixed point of $\left(\phi_{t}\right)$ with repelling spectral value $\lambda \geq-2 V(\gamma)$,

(3) or, $\tau \in \partial \mathbb{D}$ and $\lim _{t \rightarrow+\infty} \gamma(t)=\tau$. Moreover, in this case, the convergence of $\gamma$ to $\tau$ is tangential and $\left(\phi_{t}\right)$ is parabolic.

Therefore, every regular non-constant backward orbit lands at a repelling fixed point or at the Denjoy-Wolff point, and the hyperbolic step of the orbit controls the repelling spectral value of the semigroup at the fixed point. Adapting an argument from [21], one can easily prove the converse:

Proposition 3.7 Let $\left(\phi_{t}\right)$ be a semigroup, not a group, in $\mathbb{D}$. Let $\sigma \in \partial \mathbb{D}$. Assume $\sigma$ is a repelling fixed point of $\left(\phi_{t}\right)$ with repelling spectral value $\lambda \in(-\infty, 0)$. Then there exists a (non-constant) regular backward orbit $\gamma:[0,+\infty) \rightarrow \mathbb{D}$ for $\left(\phi_{t}\right)$ such that $\lim _{t \rightarrow+\infty} \gamma(t)=$ $\sigma$ and with hyperbolic step $V(\gamma)=-\frac{1}{2} \lambda$.

The previous results show that that for every repelling fixed point of a semigroup, there exists a regular backward orbit converging to such a point. Moreover, every backward orbit lands at a boundary fixed point of the semigroup. To close the circle, one might wonder whether every super-repelling fixed point is the landing point of a (non-regular) backward orbit. The answer is negative: as it is proved in [7, Prop. 4.9], a super-repelling fixed point is the landing point of a backward orbit if and only if it is not the initial point of a maximal contact arc. In particular, by [6, Prop. 4.2], if $\sigma \in \partial \mathbb{D}$ is a super-repelling fixed point for the semigroup $\left(\phi_{t}\right)$ and $G$ is the associated infinitesimal generator, then $\angle \lim _{z \rightarrow \sigma} G(z)=0$ implies that there exist no backward orbits for $\left(\phi_{t}\right)$ landing at $\sigma$.

Now we introduce the notion of pre-model:

Definition 3.8 Let $\left(\phi_{t}\right)$ be a semigroup in $\mathbb{D}$ which is not a group. Let $\sigma \in \partial \mathbb{D}$ be a repelling fixed point for $\left(\phi_{t}\right)$ with repelling spectral value $\lambda \in(-\infty, 0)$. We say that the triple $\left(\mathbb{D}, g, \eta_{t}\right)$ is a pre-model for $\left(\phi_{t}\right)$ at $\sigma$ if

(1) $\left(\eta_{t}\right)$ is the unique hyperbolic group with Denjoy-Wolff point $-\sigma$, other fixed point $\sigma$ and spectral value $-\lambda$,

(2) $g: \mathbb{D} \rightarrow \mathbb{D}$ is univalent, $\angle \lim _{z \rightarrow \sigma} g(z)=\sigma$, and $g$ is semi-conformal at $\sigma$, i.e.,

$$
\angle \lim _{z \rightarrow \sigma} \operatorname{Arg} \frac{1-\bar{\sigma} g(z)}{1-\bar{\sigma} z}=0,
$$

(3) $g \circ \eta_{t}=\phi_{t} \circ g$ for all $t \geq 0$.

Remark 3.9 If ( $\left.\mathbb{D}, g, \eta_{t}\right)$ is a pre-model for a semigroup $\left(\phi_{t}\right)$ at a repelling fixed point $\sigma \in \partial \mathbb{D}$ with repelling spectral value $\lambda \in(-\infty, 0)$, it follows that the repelling spectral value of $\left(\eta_{t}\right)$ at $\sigma$ is $\lambda$. Indeed, we have $\eta_{t}^{\prime}(\sigma)=e^{-\lambda t}$ for all $t \geq 0$. 
The proof of the next theorem can be adapted from the discrete case from [21]. We leave details to the reader.

Theorem 3.10 Let $\left(\phi_{t}\right)$ be a semigroup in $\mathbb{D}$ which is not a group. Let $\sigma \in \partial \mathbb{D}$ be a repelling fixed point for $\left(\phi_{t}\right)$ with repelling spectral value $\lambda \in(-\infty, 0)$. Then, there exists a pre-model $\left(\mathbb{D}, g, \eta_{t}\right)$ for $\left(\phi_{t}\right)$ at $\sigma$.

It can be proved directly arguing as in [21] that if $\left(\mathbb{D}, \tilde{g}, \eta_{t}\right)$ for $\left(\phi_{t}\right)$ is another pre-model, then $\tilde{g}=g \circ T$, where $T$ is a hyperbolic automorphism of $\mathbb{D}$ fixing $\pm \sigma$ (and in particular $T \circ \eta_{t}=\eta_{t} \circ T$ for all $t \geq 0$ ). This also follows easily from our further construction; see Remark 4.19.

In general, given a pre-model $\left(\mathbb{D}, g, \eta_{t}\right)$ for $\left(\phi_{t}\right)$ at $\sigma$, the map $g$ is not regular at $\sigma$ (see Sect. 8).

A straightforward consequence of Theorem 3.10 is the following:

Corollary 3.11 Let $\left(\phi_{t}\right)$ be a semigroup, not a group, in $\mathbb{D}$. Let $\sigma \in \partial \mathbb{D}$ be a repelling fixed point of $\left(\phi_{t}\right)$ with repelling spectral value $\lambda \in(-\infty, 0)$. Let $\left(\mathbb{D}, g, \eta_{t}\right)$ be a pre-model for $\left(\phi_{t}\right)$ at $\sigma$. Then, for every $\alpha \in(-\pi / 2, \pi / 2)$ there exists a (non-constant) backward orbit $\gamma:[0,+\infty) \rightarrow \mathbb{D}$ for $\left(\phi_{t}\right)$, with $\gamma([0,+\infty)) \subset g(\mathbb{D})$, converging to $\sigma$ such that

$$
\lim _{t \rightarrow+\infty} \operatorname{Arg}(1-\bar{\sigma} \gamma(t))=\alpha .
$$

Conversely, if $\gamma:[0,+\infty) \rightarrow \mathbb{D}$ is a backward orbit converging to $\sigma$, then $\gamma$ is regular if and only if it converges non-tangentially to $\sigma$. Moreover, in this case, $\gamma([0,+\infty)) \subset g(\mathbb{D})$ and there exists $\alpha \in(-\pi / 2, \pi / 2)$ such that (3.1) holds.

\section{Petals}

Definition 4.1 Let $\left(\phi_{t}\right)$ be a semigroup, not a group, in $\mathbb{D}$. Let

$$
\mathscr{W}:=\cap_{t \geq 0} \phi_{t}(\mathbb{D}) .
$$

We call $\mathscr{W}$ the backward invariant set of $\left(\phi_{t}\right)$. We call each non-empty connected component of $\stackrel{\circ}{\mathscr{W}}$, the interior of $\mathscr{W}$, a petal for $\left(\phi_{t}\right)$.

Backward invariant sets are strictly related to invariant curves. We start with a definition,

Definition 4.2 Let $\left(\phi_{t}\right)$ be a semigroup, not a group, in $\mathbb{D}$, with Denjoy-Wolff point $\tau \in \overline{\mathbb{D}}$. Let $a \in[-\infty, 0)$. A continuous curve $\gamma:(a,+\infty) \rightarrow \mathbb{D}$ is called a maximal invariant curve for $\left(\phi_{t}\right)$ if $\phi_{s}(\gamma(t))=\gamma(t+s)$ for all $s \geq 0$ and $t \in(a,+\infty), \lim _{t \rightarrow+\infty} \gamma(t)=\tau$ and there exists $p \in \partial \mathbb{D}$ such that $\lim _{t \rightarrow a^{+}} \gamma(t)=p$. We call $p$ the starting point of $\gamma$.

Remark 4.3 Let $a \in[-\infty, 0)$. Let $\gamma:(a,+\infty) \rightarrow \mathbb{D}$ be a maximal invariant curve for a semigroup $\left(\phi_{t}\right)$ which is not a group, and let $\lim _{t \rightarrow a^{+}} \gamma(t)=p \in \partial \mathbb{D}$. Then, $\gamma$ is injective. Indeed, if not, say $\gamma(t)=\gamma(s)$ for some $a<s<t$. Then $\phi_{t-s}(\gamma(s))=\gamma(t)=\gamma(s)$, implying that $\gamma(s)=\tau \in \mathbb{D}$, the Denjoy-Wolff point of $\left(\phi_{t}\right)$. But then, for all $a<t_{0}<s$, $\phi_{s-t_{0}}\left(\gamma\left(t_{0}\right)\right)=\gamma(s)=\tau$. Since $\phi_{s-t_{0}}$ is injective in $\mathbb{D}$, this forces $\gamma\left(t_{0}\right)=\tau$ for all $a<t_{0}<s$, a contradiction.

Let $h:(0,1) \rightarrow(a,+\infty)$ be any orientation preserving homeomorphism. Then, setting $\ell(0)=p, \ell(t):=\gamma(h(t))$ and $\ell(1)=\tau$, it follows that $\ell:[0,1] \rightarrow \overline{\mathbb{D}}$ is a Jordan arc (or a Jordan curve if $p=\tau$ ). 
Remark 4.4 Clearly, every non-constant backward orbit gives rise to a maximal invariant curve with starting point a fixed point by flowing forward the backward orbit and reversing orientation.

Proposition 4.5 Let $\left(\phi_{t}\right)$ be a semigroup, not a group, in $\mathbb{D}$ with Denjoy-Wolff point $\tau \in \overline{\mathbb{D}}$ and backward invariant set $\mathscr{W}$. Then, for every $z_{0} \in \mathbb{D} \backslash\{\tau\}$, there exists a unique maximal invariant curve $\gamma:(a,+\infty) \rightarrow \mathbb{D}$ for $\left(\phi_{t}\right)$ such that $\gamma(0)=z_{0}$. Moreover, $z_{0} \in \mathscr{W}$ if and only if $a=-\infty$. Also, denote by $p \in \partial \mathbb{D}$ the starting point of $\gamma$. Then,

(1) if $z_{0} \notin \mathscr{W}$ then $\gamma(t+a)=\phi_{t}(p)$ for all $t \in(0,+\infty)$, in particular $p$ is not a fixed point for $\left(\phi_{t}\right)$, and $\gamma(t) \notin \mathscr{W}$ for all $t>a$.

(2) if $z_{0} \in \mathscr{W}$ then $[0,+\infty) \ni t \mapsto \gamma(-t)$ is a backward orbit and $p$ is a boundary fixed point of $\left(\phi_{t}\right)$. Also, $z_{0} \in \stackrel{\circ}{\mathscr{W}}$ if and only if $\gamma(t) \in \stackrel{\circ}{\mathscr{W}}$ for all $t \in \mathbb{R}$.

In particular, $\phi_{t}(\stackrel{\circ}{\mathscr{W}})=\stackrel{\circ}{\mathscr{W}}, \phi_{t}(\mathscr{W} \backslash \stackrel{\circ}{\mathscr{W}})=\mathscr{W} \backslash \stackrel{\circ}{\mathscr{W}}$ and $\phi_{t}(\mathbb{D} \backslash \mathscr{W}) \subset \mathbb{D} \backslash \mathscr{W} \quad$ for all $t \geq 0$.

Proof First, we show that if there exists a maximal invariant curve $\gamma$ for $\left(\phi_{t}\right)$ such that $\gamma(0)=z_{0}$, then it is unique. Assume $\tilde{\gamma}:(\tilde{a},+\infty) \rightarrow \mathbb{D}$ is another maximal invariant curve such that $\tilde{\gamma}(0)=z_{0}$, for some $\tilde{a}<0$. Then, for $t \geq 0$,

$$
\gamma(t)=\phi_{t}(\gamma(0))=\phi_{t}\left(z_{0}\right)=\phi_{t}(\tilde{\gamma}(0))=\tilde{\gamma}(t) .
$$

For $\max \{a, \tilde{a}\}<t<0$,

$$
\phi_{-t}(\gamma(t))=\gamma(0)=z_{0}=\tilde{\gamma}(0)=\phi_{-t}(\tilde{\gamma}(t)) .
$$

Since $\phi_{-t}$ is injective, we have $\gamma(t)=\tilde{\gamma}(t)$. If $\tilde{a}>a$, then $\lim _{t \rightarrow \tilde{a}^{+}} \tilde{\gamma}(z)=\lim _{t \rightarrow \tilde{a}^{+}} \gamma(t) \in$ $\mathbb{D}$, a contradiction. Similarly, if $a<\tilde{a}$, we get a contradiction. Then, if exists, such a maximal invariant curve is unique.

Now we construct a maximal invariant curve. Let $\left(\Omega, h, \psi_{t}\right)$ be the canonical model for $\left(\phi_{t}\right)$, where $\Omega$ is either $\mathbb{C}, \mathbb{H}, \mathbb{H}^{-}$or the strip $\mathbb{S}_{\rho}$ for some $\rho>0$ and either $\psi_{t}(w)=w+i t$ or $\psi_{t}(w)=e^{-\lambda t} w$ for some $\lambda \in \mathbb{C}$ with $\operatorname{Re} \lambda>0$. Let $w_{0}:=h\left(z_{0}\right)$ and

$$
a:=\inf \left\{t<0: \psi_{-t}\left(w_{0}\right) \in h(\mathbb{D})\right\} .
$$

By the geometry of $\Omega$ (spirallike or starlike at infinity) and the form of $\psi_{t}$, it follows that $\psi_{t}\left(w_{0}\right) \in h(\mathbb{D})$ for every $t>a$. Hence, we can well define

$$
\gamma(t):=h^{-1}\left(\psi_{t}\left(w_{0}\right)\right), \quad t \in(a,+\infty) .
$$

Since $h^{-1} \circ \psi_{t}=\phi_{t} \circ h^{-1}$ on $h(\mathbb{D})$, it is easy to see that $\phi_{s}(\gamma(t))=\gamma(s+t)$ for all $s \geq 0$ and $t \in(a,+\infty)$. This implies at once that $\lim _{t \rightarrow+\infty} \gamma(t)=\tau$.

Assume that $a>-\infty$. Then, $q:=\psi_{a}\left(w_{0}\right) \in \partial h(\mathbb{D}) \cap \mathbb{C}$ and $w_{0}=\psi_{-a}(q)$. By construction, the curve $(a,+\infty) \ni \mapsto \psi_{t-a}(q)$ is contained in $h(\mathbb{D})$ and $\lim _{t \rightarrow a^{+}} \psi_{t-a}(q)=$ $q$. By [26, Lemma 2, p. 162], there exists $p \in \partial \mathbb{D}$ such that $\lim _{t \rightarrow a^{+}} h^{-1}\left(\psi_{t-a}(q)\right)=p$. Hence,

$$
\lim _{t \rightarrow a^{+}} \gamma(t)=\lim _{t \rightarrow a^{+}} h^{-1}\left(\psi_{t}\left(w_{0}\right)\right)=\lim _{t \rightarrow a^{+}} h^{-1}\left(\psi_{t}\left(\psi_{-a}(q)\right)\right)=\lim _{t \rightarrow a^{+}} h^{-1}\left(\psi_{t-a}(q)\right)=p .
$$

Moreover, for all $s \geq 0$ and $t>a$,

$$
\phi_{S}(\gamma(t))=\phi_{S}\left(h^{-1}\left(\psi_{t}\left(w_{0}\right)\right)\right)=\phi_{s}\left(h^{-1}\left(\psi_{t-a}(q)\right)\right)=h^{-1}\left(\psi_{t+s-a}(q)\right) .
$$


Hence, $\lim _{t \rightarrow a+} \phi_{s}(\gamma(t))=h^{-1}\left(\psi_{s}(q)\right) \in \mathbb{D}$. Therefore, by Lehto-Virtanen Theorem, for all $s>0$,

$$
\phi_{s}(p)=h^{-1}\left(\psi_{s}(q)\right)=h^{-1}\left(\psi_{s+a}\left(w_{0}\right)\right)=\gamma(s+a) .
$$

It is clear that $\phi_{s}(p) \notin \mathscr{W}$ for all $s>0$; hence, $\gamma(t) \notin \mathscr{W}$ for all $t>a$.

Assume now $a=-\infty$. Clearly, $[0,+\infty) \ni t \mapsto \gamma(-t)$ is a backward orbit for $\left(\phi_{t}\right)$, hence either it is constantly equal to $\tau \in \mathbb{D}$, which implies at once that $z_{0}=\tau$, against our hypothesis, or it converges to a point $p \in \partial \mathbb{D}$ which is fixed for $\left(\phi_{t}\right)$ by Proposition 3.6. Now, let $t \in \mathbb{R}$ and $s \geq 0$. Then, $\gamma(t)=\phi_{s}(\gamma(t-s))$, which implies that $\gamma(t) \in \mathscr{W}$ for all $t \in \mathbb{R}$, in particular, $z_{0} \in \mathscr{W}$.

The previous argument shows that for all $t \geq 0$

$$
\phi_{t}(\mathscr{W})=\left(\phi_{t}\right)^{-1}(\mathscr{W})=\mathscr{W} .
$$

Now, suppose $z_{0} \in \stackrel{\circ}{\mathscr{W}}$. Then, there exists an open set $U \subset \mathscr{W}$ such that $z_{0} \in U$. Let $z_{1}=\gamma\left(t_{1}\right)$ for some $t_{1} \in(a,+\infty)$. If $t_{1}>0$, then $\phi_{t_{1}}(U) \subset \mathscr{W}$ is an open neighborhood of $\phi_{t_{1}}\left(z_{0}\right)=\gamma\left(t_{1}\right)=z_{1}$; hence, $z_{1} \in \stackrel{\circ}{\mathscr{W}}$. If $t_{1}<0$, taking into account that $U \subset \phi_{t_{1}}(\mathbb{D})$, it follows that $V:=\left(\phi_{t_{1}}\right)^{-1}(U)$ is an open neighborhood of $z_{1}$. By (4.1), $V \subset \mathscr{W}$; hence $z_{1} \in \stackrel{\circ}{\mathscr{W}}$.

Remark 4.6 Let $\left(\phi_{t}\right)$ be a semigroup, not a group, in $\mathbb{D}$, with infinitesimal generator $G$. Let $\gamma:(a,+\infty) \rightarrow \mathbb{D}$ be a maximal invariant curve. Then, $\gamma$ is the maximal solution to the Cauchy problem $\frac{d}{d t} x(t)=G(x(t)), x(0)=\gamma(0)$. It follows at once differentiating in $s$ the expression $\phi_{s}(\gamma(t))=\gamma(t+s)$. Conversely, by the uniqueness property of the solution to the Cauchy problem, given $z_{0} \in \mathbb{D}$ and $\gamma:(-\epsilon,+\infty) \rightarrow \mathbb{D}, \epsilon \in(0,+\infty]$ the maximal solution to the Cauchy problem $\frac{d}{d t} x(t)=G(x(t)), x(0)=z_{0}$, then $\gamma$ is a maximal invariant curve of $\left(\phi_{t}\right)$. One can use this point of view to prove in another way the previous proposition.

Remark 4.7 Let $\left(\phi_{t}\right)$ be a semigroup, not a group, in $\mathbb{D}$. Let $z_{0} \in \mathbb{D} \backslash\{\tau\}$ and let $\gamma$ : $(a,+\infty) \rightarrow \mathbb{D}$ be the unique maximal invariant curve for $\left(\phi_{t}\right)$ such that $\gamma(0)=z_{0}, a<0$. Let $t_{0} \in(a,+\infty)$. It is easy to check that $\tilde{\gamma}:\left(a+t_{0},+\infty\right) \rightarrow \mathbb{D}$ (where, if $a=-\infty$, we set $\left.a+t_{0}=-\infty\right)$ is a maximal invariant curve for $\left(\phi_{t}\right)$ such that $\tilde{\gamma}(0)=\gamma\left(t_{0}\right)$. In other words, for every $z \in \gamma((a,+\infty))$, the image of the maximal invariant curve for $\left(\phi_{t}\right)$ which values $z$ at time 0 are $\gamma((a,+\infty))$.

Remark 4.8 The uniqueness of a maximal invariant curve holds also at the starting point in case this is not a fixed point. More precisely, let $\left(\phi_{t}\right)$ be a semigroup, not a group. Let $\gamma:(a,+\infty) \rightarrow \mathbb{D}$ be a maximal invariant curve for $\left(\phi_{t}\right)$ with $a<0$ and starting point $\sigma \in \partial \mathbb{D}$. Assume $a>-\infty$. If $\tilde{\gamma}:(\tilde{a},+\infty) \rightarrow \mathbb{D}$ is a maximal invariant curve for $\left(\phi_{t}\right)$, $\tilde{a}<0$, such that $\lim _{t \rightarrow \tilde{a}^{+}} \tilde{\gamma}(t)=\sigma$, then $\tilde{a}>-\infty$ and $\gamma((a,+\infty))=\tilde{\gamma}((\tilde{a},+\infty))$.

Indeed, by Proposition 4.5, $a>-\infty$ implies $\sigma$ is not a fixed point of $\left(\phi_{t}\right)$ which in turn, by the same token, implies $\tilde{a}>-\infty$. Then, by Proposition 4.5(1), $\gamma(t+a)=\phi_{t}(\sigma)=\tilde{\gamma}(t+\tilde{a})$ for all $t \geq 0$, from which the previous statement follows at once.

Now, we turn our attention to petals:

Proposition 4.9 Let $\left(\phi_{t}\right)$ be a semigroup, not a group, in $\mathbb{D}$ with Denjoy-Wolff point $\tau \in \overline{\mathbb{D}}$. Let $\Delta$ be a petal for $\left(\phi_{t}\right)$. Then,

(1) $\Delta$ is simply connected, 
(2) $\phi_{t}(\Delta)=\Delta$ for all $t \geq 0$ and $\left(\left.\phi_{t}\right|_{\Delta}\right)$ is a continuous group of automorphisms of $\Delta$,

(3) $\tau \in \partial \Delta$,

(4) there exists $\sigma \in \partial \mathbb{D} \cap \partial \Delta$ (possibly $\sigma=\tau$ ) such for every $z \in \Delta$ the curve $[0,+\infty) \ni$ $t \mapsto\left(\left.\phi_{t}\right|_{\Delta}\right)^{-1}(z)$ is a regular backward orbit for $\left(\phi_{t}\right)$ which converges to $\sigma$. Moreover, $\sigma$ is a boundary regular fixed point and if $\sigma=\tau$, then $\left(\phi_{t}\right)$ is parabolic.

Proof (1) Seeking for a contradiction, we assume $\Delta$ is not simply connected. Hence, there exists a Jordan curve $\Gamma \subset \Delta$ such that the bounded connected component of $\mathbb{C} \backslash \Gamma$ contains a point $z_{0} \notin \mathscr{W}$, the backward invariant set of $\left(\phi_{t}\right)$. By Proposition 4.5 , there exists a maximal invariant curve $\gamma:(a,+\infty) \rightarrow \mathbb{D}$ such that $\lim _{z \rightarrow a^{-}} \gamma(t)=p \in \partial \mathbb{D}$ and $\gamma(0)=z_{0}$. Moreover, $\gamma(t) \notin \mathscr{W}$ for all $t>a$. But $\gamma((a,+\infty))$ has to intersect $\Gamma$, a contradiction. Therefore, $\Delta$ is simply connected.

(2) By Proposition 4.5, $\phi_{t}(\Delta) \subset \stackrel{\circ}{\mathscr{W}}$ for all $t \geq 0$. Hence, since $\Delta$ is connected, if $z_{0} \in \Delta$ then $[0,+\infty) \ni t \mapsto \phi_{t}\left(z_{0}\right)$ is contained in $\Delta$. That is, $\phi_{t}(\Delta) \subseteq \Delta$ for all $t \geq 0$. On the other hand, if $z_{0} \in \Delta \backslash\{\tau\}$, let $\gamma:(-\infty,+\infty) \rightarrow \Delta$ be the maximal invariant curve such that $\gamma(0)=z_{0}$, given by Proposition 4.5. Hence, since $\Delta$ is connected and $\gamma((-\infty,+\infty)) \subset \stackrel{\circ}{\mathscr{W}}$, it follows that $\gamma(t) \in \Delta$ for all $t \in \mathbb{R}$. Thus, $\phi_{t}(\gamma(t))=\gamma(0)=z_{0}$ for every $t \geq 0$. Namely, $\phi_{t}(\Delta)=\Delta$ for all $t \geq 0$. Therefore, $\left(\left.\phi_{t}\right|_{\Delta}\right)$ is a continuous semigroup whose iterates are automorphisms of $\Delta$. Clearly, it extends to a continuous group $\left(\left.\phi_{t}\right|_{\Delta}\right)$ of $\Delta$.

(3) If $z \in \Delta$, then $\phi_{t}(z) \in \Delta$ for all $t \geq 0$ and $\phi_{t}(z) \rightarrow \tau$. Hence, $\tau \in \bar{\Delta}$. In particular, if $\left(\phi_{t}\right)$ is not elliptic, then $\tau \in \partial \Delta$. Assume $\left(\phi_{t}\right)$ is elliptic and $\tau \in \Delta$. Since $\Delta$ is simply connected, there exists a univalent map $f: \mathbb{D} \rightarrow \mathbb{C}$ such that $f(\mathbb{D})=\Delta$ and $f(0)=\tau$. Hence, by (2), $\left(f^{-1} \circ \phi_{t} \circ f\right)$ is a group of $\mathbb{D}$ with Denjoy-Wolff point 0 such that

$$
1=\left|\left(f^{-1} \circ \phi_{t} \circ f\right)^{\prime}(0)\right|=\left|\phi_{t}^{\prime}(\tau)\right| .
$$

By the Schwarz Lemma, it follows that $\left(\phi_{t}\right)$ is a group, against our assumption.

(4) Let $z \in \Delta$. Taking into account that $\left.\phi_{t}\right|_{\Delta}$ is an automorphism of $\Delta$ for all $t \geq 0$, and denoting by $k_{\Delta}$ the hyperbolic distance in $\Delta$, we have

$$
\begin{aligned}
\omega\left(\left(\left.\phi_{t}\right|_{\Delta}\right)^{-1}(z),\left(\left.\phi_{t+1}\right|_{\Delta}\right)^{-1}(z)\right) & \leq k_{\Delta}\left(\left(\left.\phi_{t}\right|_{\Delta}\right)^{-1}(z),\left(\left.\phi_{t+1}\right|_{\Delta}\right)^{-1}(z)\right) \\
& =k_{\Delta}\left(z,\left(\left.\phi_{1}\right|_{\Delta}\right)^{-1}(z)\right)<+\infty .
\end{aligned}
$$

Moreover, $\phi_{s}\left(\left(\left.\phi_{t}\right|_{\Delta}\right)^{-1}(z)\right)=\left(\left.\phi_{t-s}\right|_{\Delta}\right)^{-1}(z)$ for all $0 \leq s \leq t$. Therefore, $[0,+\infty) \ni$ $t \mapsto\left(\left.\phi_{t}\right|_{\Delta}\right)^{-1}(z)$ is a regular backward orbit for $\left(\phi_{t}\right)$. Hence, by Proposition 3.6, there exists $\sigma \in \partial \mathbb{D} \cap \partial \Delta$ such that $\lim _{t \rightarrow+\infty}\left(\left.\phi_{t}\right|_{\Delta}\right)^{-1}(z)=\sigma$-and, if $\sigma=\tau$, then $\left(\phi_{t}\right)$ is parabolic.

Finally, if $w \in \Delta$, for all $t \geq 0$,

$$
\omega\left(\left(\left.\phi_{t}\right|_{\Delta}\right)^{-1}(z),\left(\left.\phi_{t}\right|_{\Delta}\right)^{-1}(w)\right) \leq k_{\Delta}\left(\left(\left.\phi_{t}\right|_{\Delta}\right)^{-1}(z),\left(\left.\phi_{t}\right|_{\Delta}\right)^{-1}(w)\right)=k_{\Delta}(z, w)<+\infty,
$$

hence $\lim _{t \rightarrow+\infty}\left(\left.\phi_{t}\right|_{\Delta}\right)^{-1}(w)=\sigma$.

The previous result allows to give the following definition:

Definition 4.10 Let $\left(\phi_{t}\right)$ be a semigroup, not a group, in $\mathbb{D}$ with Denjoy-Wolff point $\tau \in \overline{\mathbb{D}}$. Let $\Delta$ be a petal for $\left(\phi_{t}\right)$. We say that $\Delta$ is a hyperbolic petal if $\partial \Delta$ contains a repelling fixed point of $\left(\phi_{t}\right)$. Otherwise, we call $\Delta$ a parabolic petal.

Remark 4.11 By Proposition 4.9, a petal contains no (inner) fixed points of the semigroup. Moreover, only parabolic semigroup can have parabolic petals. 
Our aim now is to describe the boundary of petals. We start with the following result which, taking into account Remark 4.4, shows that backward orbits which land at the Denjoy-Wolff point have to be contained in the closure of a parabolic petal:

Proposition 4.12 Let $\left(\phi_{t}\right)$ be a non-elliptic semigroup in $\mathbb{D}$ with Denjoy-Wolff point $\tau \in \partial \mathbb{D}$. Let $\gamma:(-\infty,+\infty) \rightarrow \mathbb{D}$ be a maximal invariant curve for $\left(\phi_{t}\right)$ such that $\lim _{t \rightarrow-\infty} \gamma(t)=\tau$. Let $J$ be the Jordan curve defined by $\gamma$, i.e., $J:=\overline{\gamma(\mathbb{R})}$. Let $V$ be the bounded connected component of $\mathbb{C} \backslash J$. Then there exists a parabolic petal $\Delta$ of $\left(\phi_{t}\right)$ such that $V \subseteq \Delta$. In particular, $J$ is contained in the closure of a parabolic petal of $\left(\phi_{t}\right)$ and the semigroup is parabolic.

Proof By Remark 4.3, $J$ is a Jordan curve. Fix $\zeta_{0} \in V$. Let $\eta:(a,+\infty) \rightarrow \mathbb{D}$ be the maximal invariant curve for $\left(\phi_{t}\right)$ such that $\eta(0)=\zeta_{0}, a<0$. Note that since $J$ is the closure of the image of a maximal invariant curve which does not contain $\zeta_{0}$, we have $\eta((a,+\infty)) \cap J=\emptyset$ by Remark 4.7. Hence, $\eta((a,+\infty)) \subset V$. By Proposition 4.5, the initial point $w_{0}$ of $\eta$ belongs to $\partial \mathbb{D}$; hence, the only possibility is $w_{0}=\tau$. In this case, again by Proposition 4.5 , we have $\zeta_{0} \in \mathscr{W}$, the backward invariant set of $\left(\phi_{t}\right)$. By the arbitrariness of $\zeta_{0}$, this implies

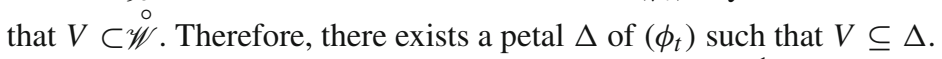

Finally, let $z \in V$. The curve $[0,+\infty) \ni t \mapsto\left(\left.\phi_{t}\right|_{\Delta}\right)^{-1}(z)$ is a backward orbit of $\left(\phi_{t}\right)$ by Proposition 4.9. By Remark 4.4, it extends to a maximal invariant curve of $\left(\phi_{t}\right)$. Hence, for what we already proved, such a curve converges to $\tau$. It follows that $\Delta$ is parabolic, again by Proposition 4.9(4).

Now, we focus on the boundary of petals. To this aim, and for the subsequent results, we need a lemma:

Lemma 4.13 Let $\left(\phi_{t}\right)$ be a semigroup, not a group, in $\mathbb{D}$ with Denjoy-Wolff point $\tau \in \overline{\mathbb{D}}$. Suppose $\Delta \subset \mathbb{D}$ is a petal and $z_{0} \in \partial \Delta \cap(\mathbb{D} \backslash\{\tau\})$. Let $\gamma:(a,+\infty) \rightarrow \mathbb{D}, a<0$, be the maximal invariant curve of $\left(\phi_{t}\right)$ such that $\gamma(0)=z_{0}$. Then, $\gamma((a,+\infty)) \subset \partial \Delta$.

Proof Let $\mathscr{W}$ be the backward invariant set of $\left(\phi_{t}\right)$. Since $z_{0} \in \partial \Delta$, it follows that $z_{0} \notin \stackrel{\circ}{\mathscr{W}}$. By Proposition 4.5, $\gamma(t) \notin \stackrel{\circ}{\mathscr{W}}$ for all $t \in(a,+\infty)$. In particular, $\gamma(t) \notin \Delta$ for all $t \in(a,+\infty)$.

Let $t \geq 0$ and fix $\delta>0$. Since $z_{0} \in \partial \Delta$, there exists $w \in \Delta$ such that $\omega\left(z_{0}, w\right)<\delta$. Then,

$$
\omega\left(\phi_{t}(w), \gamma(t)\right)=\omega\left(\phi_{t}(w), \phi_{t}\left(z_{0}\right)\right) \leq \omega\left(w, z_{0}\right)<\delta .
$$

Since $\phi_{t}(w) \in \Delta$ for all $t \geq 0$ by Proposition 4.9, by the arbitrariness of $\delta$, we have $\gamma(t) \in \partial \Delta$ for all $t \geq 0$. Now, let $t \in(a, 0)$. Assume by contradiction that $\gamma(t) \notin \partial \Delta$. Then, there exists an open neighborhood $U$ of $\gamma(t)$ such that $U \cap \Delta=\emptyset$. Since $\phi_{-t}(\gamma(t))=\gamma(0)=z_{0}$, and $\phi_{-t}(U)$ is open, there exists $w \in \phi_{-t}(U) \cap \Delta$. Since $\left.\phi_{-t}\right|_{\Delta}$ is an automorphism of $\Delta$, it follows that $\left(\left.\phi_{-t}\right|_{\Delta}\right)^{-1}(w) \in \Delta$. But $\phi_{-t}$ is injective, hence $\left(\left.\phi_{-t}\right|_{\Delta}\right)^{-1}(w) \in U$, a contradiction. Hence, $\gamma(t) \in \partial \Delta$ for all $t \in(a,+\infty)$.

We first consider the case in which the boundary of a petal contains a maximal invariant curve starting at the Denjoy-Wolff point:

Proposition 4.14 Let $\left(\phi_{t}\right)$ be a parabolic semigroup in $\mathbb{D}$ with Denjoy-Wolffpoint $\tau \in \partial \mathbb{D}$. Let $\Delta \subset \mathbb{D}$ be a petal of $\left(\phi_{t}\right)$. Suppose there exists a maximal invariant curve $\gamma:(-\infty,+\infty) \rightarrow$ $\mathbb{D}$ of $\left(\phi_{t}\right)$ such that $\lim _{t \rightarrow-\infty} \gamma(t)=\tau$. Let $J:=\overline{\gamma(\mathbb{R})}$ (a Jordan curve), and let $V$ be the 
bounded connected component of $\mathbb{C} \backslash J$. Assume that $J \cap \partial \Delta \cap \mathbb{D} \neq \emptyset$. Then, $\Delta$ is parabolic, $\Delta=V$ and $J=\partial \Delta$.

In particular, the boundary of a hyperbolic petal cannot contain maximal invariant curves with starting point $\tau$.

Proof By Lemma 4.13, $J \subset \partial \Delta$. Since $\Delta$ is connected and $J \cap \Delta=\emptyset$, either $\Delta \subseteq V$ or $\Delta \subseteq \mathbb{D} \backslash \bar{V}$. But, by Proposition 4.12, $V$ is contained in a parabolic petal. Therefore, if $\Delta \subset V$, then, in fact, $V=\Delta$ and the statement holds.

Thus, assume by contradiction that $\Delta \subseteq U:=\mathbb{D} \backslash \bar{V}$. Let $z_{0} \in J \cap \mathbb{D}$. We claim that there exists an open neighborhood $A$ of $z_{0}$ such that $A \cap U \subset \Delta$.

Assume the claim is true. Let $\mathscr{W}$ be the backward invariant set of $\left(\phi_{t}\right)$. Note that $J \cap \mathbb{D} \subset$

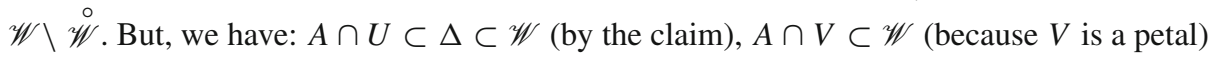
and $J \cap \mathbb{D} \subset \mathscr{W}$ (by Proposition 4.5). Hence, $A \subset \mathscr{W}$, which implies $z_{0} \in \stackrel{\circ}{\mathscr{W}}$, a contradiction.

We are left to prove the claim.

If $\Delta=U$, then set $A=U$.

Otherwise, there exists $\zeta_{0} \in \partial \Delta \cap U$. Let $\eta_{0}:(a,+\infty) \rightarrow \mathbb{D}, a<0$, be the maximal invariant curve such that $\eta_{0}(0)=\zeta_{0}$. Let $C_{0}$ be its closure (it is a Jordan curve by Remark 4.3). By Lemma 4.13, $C_{0} \subset \partial \Delta$. The Jordan curve $C_{0}$ divides $\mathbb{D}$ in two connected components, one of them, call it $B_{0}$, contains $\Delta$. Since $\zeta_{0} \notin J$, we have $C_{0} \cap J \cap \mathbb{D}=\emptyset$ by Remark 4.7, and hence, $\bar{V} \cap \mathbb{D} \subset B_{0}$.

Let $p_{0}$ be the starting point of $\eta_{0}$. If $p_{0}=\tau$, then $B_{0}$ is the bounded connected component of $\mathbb{D} \backslash C_{0}$; hence, it is contained in a parabolic petal by Proposition 4.12. This implies $J \cap$ $\mathbb{D} \subset \stackrel{\mathscr{W}}{ }$, a contradiction. Therefore, $p_{0} \neq \tau$.

If $B_{0}=\Delta$, we are done setting $A=B_{0}$. Otherwise, there exists $\zeta_{1} \in \partial \Delta \cap B_{0}$, and, arguing as before, we obtain another Jordan curve $C_{1} \subset \partial \Delta$, which does not intersect $C_{0} \cap \mathbb{D}$ and $J \cap \mathbb{D}$. Let $B_{1}$ be the connected component of $\mathbb{D} \backslash C_{1}$ which contains $\Delta$. By construction, such connected component contains $\bar{V} \cap \mathbb{D}$ and $C_{0} \cap \mathbb{D}$.

Let $p_{1}$ be the starting point of the maximal invariant curve whose closure defines $C_{1}$. As before, $p_{1} \neq \tau$. By construction, $\Delta \subset G:=B_{0} \cap B_{1} \cap U$. If $G=\Delta$, we set $A=G$ and we are done.

Otherwise, note that, by construction, $G$ is a simply connected domain whose boundary is given by $J \cup C_{0} \cup C_{1} \cup T$, where $T$ is a closed arc in $\partial \mathbb{D}$ with end points $p_{0}$ and $p_{1}$ (we set $T=\left\{p_{0}\right\}$ in case $p_{0}=p_{1}$ ). If there were a point $\zeta_{2} \in G \cap \partial \Delta$, the Jordan curve $C_{2}$, defined as before by $\zeta_{2}$, would divide $G$ into two connected components and $\Delta$ would belong to one of the two connected components. But this is impossible because $J, C_{0}, C_{1} \subset \partial \Delta$. The claim is proved.

Now we are in good shape to describe the boundary of petals:

Proposition 4.15 Let $\left(\phi_{t}\right)$ be a semigroup, not a group, in $\mathbb{D}$ with Denjoy-Wolff point $\tau \in \overline{\mathbb{D}}$. Let $\Delta$ be a petal for $\left(\phi_{t}\right)$. Then, one and only one of the following cases can happen:

(1) $\tau \in \mathbb{D}$ and there exists a Jordan arc $J$ with end points $\tau$ and a point $p \in \partial \mathbb{D}$ such that $\partial \Delta=J \cup \partial \mathbb{D}$. In particular, in this case, $\Delta$ is the only petal of $\left(\phi_{t}\right)$;

(2) $\tau \in \partial \mathbb{D}$ and there exists a Jordan arc $J$ with end points $\tau$ and a point $p \in \partial \mathbb{D} \backslash\{\tau\}$ such that $\partial \Delta=J \cup A$ where $A$ is an arc in $\partial \mathbb{D}$ with end points $\tau$ and $p$;

(3) there exist two Jordan arcs $J_{1}, J_{2}$ with end points $p \in \partial \mathbb{D} \backslash\{\tau\}$ and $\tau$ such that $J_{1} \cap J_{2}=$ $\{\tau, p\}$ and $\partial \Delta=J_{1} \cup J_{2}$; 
(4) there exist two Jordan arcs $J_{1}, J_{2}$ such that $J_{j}$ has end points $\tau$ and $p_{j} \in \partial \mathbb{D} \backslash\{\tau\}$, $j=1,2$, with $J_{1} \cap J_{2}=\{\tau\}$ and $\partial \Delta=J_{1} \cup J_{2} \cup A$, where $A \subset \partial \mathbb{D}$ is a closed arc with end points $p_{1}$ and $p_{2}$;

(5) $\tau \in \partial \mathbb{D}$ and there exists a Jordan curve $J$ such that $J \cap \partial \mathbb{D}=\{\tau\}, \partial \Delta=J$ and $\Delta$ is the bounded connected component of $\mathbb{C} \backslash J$. In this case, $\Delta$ is a parabolic petal.

In particular, $\partial \Delta$ is locally connected and, in cases (2), (3), (4) and (5), $\Delta$ is a Jordan domain.

Proof Since $\left(\phi_{t}\right)$ is not a group by hypothesis, it follows that $\Delta \neq \mathbb{D}$ by Proposition 4.9. Hence, $\partial \Delta \cap \mathbb{D} \neq \emptyset$. Since $\Delta$ is simply connected, $\partial \Delta \cap \mathbb{D}$ cannot reduce to one point. Let $z_{0} \in \partial \Delta \cap \mathbb{D} \backslash\{\tau\}$.

Let $\gamma:(a,+\infty) \rightarrow \mathbb{D}$ be the maximal invariant curve such that $\gamma(0)=z_{0}, a<0$, given by Proposition 4.5. Let $J$ be the closure of $\gamma((a,+\infty))$. By Remark 4.3, $J$ is a Jordan arc with end points $\tau$ and $p \in \partial \mathbb{D}$ (if $p=\tau$, then $J$ is a Jordan curve). By Lemma 4.13, $J \subset \partial \Delta$.

By Proposition 4.14, if $p=\tau$, then we are in case (5).

If $\partial \Delta \backslash J \subset \partial \mathbb{D}$ and $p \neq \tau$, then we are in case (1) or (2). In this case, if $\tau \in \mathbb{D}$, then $\partial \Delta$ is necessarily the union of $J$ with $\partial \mathbb{D}$ and, moreover, $\mathbb{D} \backslash \Delta=J$, which, since $J$ has no interior, implies that $\Delta$ is the only petal of $\left(\phi_{t}\right)$. On the other hand, if $\tau \in \partial \mathbb{D}$, and $p \neq \tau$, since $\Delta$ is connected, and $J$ disconnects $\mathbb{D}$ in two connected components by Jordan's Theorem, it follows that $\partial \Delta$ is the union of $J$ with an arc in $\partial \mathbb{D}$ with end points $\tau$ and $p$.

Now, assume there exists $z_{0}^{\prime} \in \partial \Delta \backslash J \cap \mathbb{D}$. We repeat the above argument, in order to obtain another Jordan arc (or Jordan curve) $J_{2} \subset \partial \Delta$ which contains $z_{0}^{\prime}$ and $J_{2}$ is contained in $\mathbb{D}$ except, at most, the two end points. By Remark 4.7, since the interior parts of both $J$ and $J_{2}$ are maximal invariant curves, and $z_{0}^{\prime} \notin J$, it follows that $J \cap J_{2} \subset \partial \mathbb{D} \cup\{\tau\}$ (that is, $J$ and $J_{2}$ can have in common only the end points). Let $p_{2} \in \partial \mathbb{D}$ be the initial point of $J_{2}$. By Proposition 4.14, $p_{2} \neq \tau$.

We claim that $\left(\partial \Delta \backslash\left(J \cup J_{2}\right)\right) \subset \partial \mathbb{D}$. Indeed, if this is not the case, one can find a point $w_{0} \in\left(\partial \Delta \backslash\left(J \cup J_{2}\right)\right) \cap \mathbb{D}$. Repeating the above argument, we end up with another Jordan curve $J_{3}$ whose interior does not intersect $J, J_{2}$. Recalling that $J, J_{2}, J_{3}$ have a common end point $\tau$ and another end point on $\partial \mathbb{D} \backslash\{\tau\}$, it is easy to see that we reach a contradiction. For instance, in case $\tau \in \mathbb{D}$, the Jordan curve $J \cup J_{2}$ divides $\mathbb{D}$ into two connected components, and $\Delta$ has to stay in one of them, call it $U$. Then, $J_{3}$ is contained in $U$ and divides $U$ into two connected components, one whose boundary is $J, J_{3}$ and (possibly) an arc on $\partial \mathbb{D}$, and the other whose boundary is $J, J_{2}$ and (possibly) an arc on $\partial \mathbb{D}$. Since $\Delta$ is connected, it has to stay in one of the two components, say the first, but then $J_{2}$ cannot be contained in $\partial \Delta$, a contradiction. The other cases are similar.

If $J_{1}$ and $J_{2}$ have the same end points, then we are in case (3). If $J_{1}$ has a different end point than $J$ (they both have $\tau$ as common end point), then we are in case (4).

Every case given by Proposition 4.15 actually happens, as we will see in Sect. 7 .

Proposition 4.16 Let $\left(\phi_{t}\right)$ be a semigroup, not a group, in $\mathbb{D}$ with Denjoy-Wolff point $\tau \in \overline{\mathbb{D}}$. Let $\Delta$ be a petal for $\left(\phi_{t}\right)$.

(1) If $\Delta$ is a hyperbolic petal, then there exists a repelling fixed point $\sigma \in \partial \Delta$ of $\left(\phi_{t}\right)$ such that $\partial \Delta \backslash\{\tau, \sigma\}$ does not contain any (repelling or super-repelling) boundary fixed point of $\left(\phi_{t}\right)$.

(2) If $\Delta$ is a parabolic petal, then $\partial \Delta \backslash\{\tau\}$ does not contain any (repelling or super-repelling) boundary fixed point of $\left(\phi_{t}\right)$. 
Proof Since $\Delta$ is simply connected by Proposition 4.9, there exists a univalent function $g: \mathbb{D} \rightarrow \mathbb{C}$ such that $g(\mathbb{D})=\Delta$. Let $\psi_{t}:=g^{-1} \circ \phi_{t} \circ g, t \geq 0$. Since $\left(\left.\phi_{t}\right|_{\Delta}\right)$ is a continuous group of automorphisms of $\Delta$ by Proposition 4.9 , it follows that $\left(\psi_{t}\right)$ is a group in $\mathbb{D}$. Moreover, $\lim _{t \rightarrow+\infty} \phi_{t}(g(0)) \rightarrow \tau \in \partial \Delta$, and hence $\psi_{t}(0)=g^{-1}\left(\phi_{t}(g(0))\right)$ can accumulate only on $\partial \mathbb{D}$. Therefore, $\left(\psi_{t}\right)$ is a non-elliptic group in $\mathbb{D}$.

By Proposition 4.15, $\partial \Delta$ is locally connected, hence, by Carathéodory Extension Theorem, $g$ extends to a continuous and surjective function, which we still denote by $g$, from $\overline{\mathbb{D}}$ to $\bar{\Delta}$. In particular, for all $p \in \partial \mathbb{D}$,

$$
g\left(\psi_{t}(p)\right)=\lim _{r \rightarrow 1^{-}} g\left(\psi_{t}((1-r) p)=\lim _{r \rightarrow 1^{-}} \phi_{t}(g((1-r) p)) .\right.
$$

By Lehto-Virtanen Theorem, it follows that the non-tangential limit of $\phi_{t}$ at $g(p)$ is $g\left(\psi_{t}(p)\right)$, that is,

$$
g\left(\psi_{t}(p)\right)=\phi_{t}(g(p)) \quad \forall p \in \partial \mathbb{D}, \quad t \geq 0 .
$$

Let $p \in \partial \mathbb{D}$ be such that $g(p)$ is a fixed point of $\left(\phi_{t}\right)$. Hence, by (4.2), $g\left(\psi_{t}(p)\right)=g(p)$ for all $t \geq 0$. We claim that this implies that $\psi_{t}(p)=p$ for all $t \geq 0$. Otherwise, the image $[0,+\infty) \ni t \mapsto \psi_{t}(p)$ would be an arc in $\partial \mathbb{D}$ where $g$ is constant, a contradiction.

Since $\left(\psi_{t}\right)$ is a non-elliptic group in $\mathbb{D}$, it has at most two fixed points on $\partial \mathbb{D}$; hence, there exist at most two fixed points for $\left(\phi_{t}\right)$ on $\partial \Delta$. From this, (1) follows at once.

Now, assume that $\Delta$ is parabolic. By Remark 4.11, $\left(\phi_{t}\right)$ is necessarily parabolic, and $\tau \in$ $\partial \mathbb{D}$. Hence, by Proposition 4.15, $\Delta$ is a Jordan domain and $g: \overline{\mathbb{D}} \rightarrow \bar{\Delta}$ is a homeomorphism. Now, by Proposition 4.9(4), there exists a regular backward orbit $\gamma:[0,+\infty) \rightarrow \Delta$ such that $\lim _{t \rightarrow+\infty} \gamma(t)=\tau$. It is easy to see that $g^{-1} \circ \gamma$ is a backward orbit for $\left(\psi_{t}\right)$ which converges to $g^{-1}(\tau)$. Since $\psi_{t}(0)=g^{-1}\left(\phi_{t}(g(0))\right) \rightarrow g^{-1}(\tau)$, it follows that $\left(\psi_{t}\right)$ has a backward orbit which converges to its Denjoy-Wolff point. Hence, $\left(\psi_{t}\right)$ is a parabolic group by Proposition 3.3. Therefore, $\left(\psi_{t}\right)$ has only one fixed point on $\partial \mathbb{D}$, and so $\left(\phi_{t}\right)$ has a unique fixed point on $\partial \Delta$ which is $\tau$.

As a direct consequence of Proposition 4.16, we have:

Corollary 4.17 Let $\left(\phi_{t}\right)$ be a semigroup, not a group, in $\mathbb{D}$ with Denjoy-Wolff point $\tau \in \overline{\mathbb{D}}$. Suppose $\Delta$ is a petal of $\left(\phi_{t}\right)$. Let $J \subset \partial \Delta \cap(\mathbb{D} \backslash\{\tau\})$ be a connected component. Then, the closure of $J$ is a Jordan arc (or Jordan curve) with end points $\tau$ and $p_{0} \in \partial \mathbb{D}$. Moreover,

(1) if $\Delta$ is hyperbolic and $\sigma \in \partial \Delta$ is the unique (repelling) fixed point of $\left(\phi_{t}\right)$ contained in $\bar{\Delta}$, then either $p_{0}=\sigma$ or $p_{0}$ is not a fixed point of $\left(\phi_{t}\right)$.

(2) if $\Delta$ is parabolic, then either $p_{0}=\tau$ or $p_{0}$ is not a fixed point of $\left(\phi_{t}\right)$.

The previous results show that the closure of every hyperbolic petal contains exactly one repelling fixed point; now we show the converse:

Proposition 4.18 Let $\left(\phi_{t}\right)$ be a semigroup, not a group, in $\mathbb{D}$ with Denjoy-Wolff point $\tau \in \overline{\mathbb{D}}$. Suppose $\sigma \in \partial \mathbb{D}$ is a repelling fixed point for $\left(\phi_{t}\right)$. Then, there exists a unique hyperbolic petal $\Delta$ such that $\sigma \in \partial \Delta$. Moreover, for all $M>1$ there exists $\epsilon>0$ such that

$$
S(\sigma, M) \cap\{\zeta \in \mathbb{C}:|\zeta-\sigma|<\epsilon\} \subset \Delta,
$$

where $S(\sigma, M):=\{z \in \mathbb{D}:|\sigma-z|<M(1-|z|)\}$ is a Stolz region in $\mathbb{D}$.

Also, if $\left(\mathbb{D}, g, \eta_{t}\right)$ is a pre-model for $\left(\phi_{t}\right)$ at $\sigma$ then $g(\mathbb{D})=\Delta$. 
Proof By Theorem 3.10, there exists a pre-model $\left(\mathbb{D}, g, \eta_{t}\right)$ for $\left(\phi_{t}\right)$ at $\sigma$. For all $t \geq 0$,

$$
\phi_{t}(g(\mathbb{D}))=g\left(\eta_{t}(\mathbb{D})\right)=g(\mathbb{D}) .
$$

This implies that $g(\mathbb{D}) \subset \mathscr{W}$, the backward invariant set of $\left(\phi_{t}\right)$. Since $g(\mathbb{D})$ is open and simply connected, there exists a petal $\Delta$ such that $g(\mathbb{D}) \subset \Delta$. Moreover, $\eta_{-t}(0)$ converges non-tangentially to $\sigma$ as $t \rightarrow+\infty$ by Proposition 3.3, hence, since $\angle \lim _{z \rightarrow \sigma} g(z)=\sigma$, we have

$$
\lim _{t \rightarrow+\infty} g\left(\eta_{-t}(0)\right)=\sigma
$$

that is, $\sigma \in \overline{g(\mathbb{D})}$. Since $\Delta$ contains no fixed points of $\left(\phi_{t}\right)$ by Remark 4.11, it follows that $\sigma \in \partial \Delta$, proving the first part of the statement.

Now we show that $g(\mathbb{D})=\Delta$. Let $z_{0} \in \Delta$. By Proposition 4.9, the curve $[0,+\infty) \ni t \mapsto$ $\left(\left.\phi_{t}\right|_{\Delta}\right)^{-1}\left(z_{0}\right)$ is a regular backward orbit for $\left(\phi_{t}\right)$ which converges to $\sigma$, and, by Proposition 3.6, the convergence to $\sigma$ is non-tangential. Therefore, by Corollary $3.11,\left(\left.\phi_{t}\right|_{\Delta}\right)^{-1}\left(z_{0}\right) \in$ $g(\mathbb{D})$ for all $t \geq 0$. In particular, $z_{0} \in g(\mathbb{D})$ and hence $\Delta=g(\mathbb{D})$ by the arbitrariness of $z_{0}$. Moreover, since $g$ is semi-conformal at $\sigma$, for all $M>1$ there exists $\epsilon>0$ such that

$$
S(\sigma, M) \cap\{\zeta \in \mathbb{C}:|\zeta-\sigma|<\epsilon\} \subset g(\mathbb{D})=\Delta .
$$

Finally, we are left to show that $\Delta$ is the unique petal which contains $\sigma$ on its boundary. Assume by contradiction this is not the case, and let $\Delta^{\prime}$ be a petal different from $\Delta$ such that $\sigma \in \partial \Delta^{\prime}$. Note that $\Delta \cap \Delta^{\prime}=\emptyset$ (since they are different open connected components of the interior of the backward invariant set of $\left(\phi_{t}\right)$ ). We claim that $\Delta^{\prime}$ has to be a Jordan domain. Indeed, looking at Proposition 4.15, we see if $\Delta^{\prime}$ is not a Jordan domain, then $\Delta^{\prime}$ is the only petal of $\left(\phi_{t}\right)$, forcing $\Delta=\Delta^{\prime}$. Let $f: \mathbb{D} \rightarrow \mathbb{C}$ be univalent such that $f(\mathbb{D})=\Delta^{\prime}$. By Carathéodory's extension theorem, $f$ extends as a homeomorphism-which we still denote by $f$-from $\overline{\mathbb{D}}$ to $\overline{\Delta^{\prime}}$. By Proposition $4.9, \tau \in \partial \Delta^{\prime}$ and $\left(\left.\phi_{t}\right|_{\Delta^{\prime}}\right)$ is a continuous group of automorphisms of $\Delta^{\prime}$. Hence, arguing as in the proof of Proposition 4.16, it is easy to see that $\left(f^{-1} \circ \phi_{t} \circ f\right)$ is a group in $\mathbb{D}$, with fixed points $f^{-1}(\tau)$ and $f^{-1}(\sigma)$. Therefore, $\left(f^{-1} \circ \phi_{t} \circ f\right)$ is a hyperbolic group and by Remark 3.4, it has a regular backward orbit $\gamma$ converging to $f^{-1}(\sigma)$. It is easy to see that $f \circ \gamma$ is a backward orbit for $\left(\phi_{t}\right)$ converging to $\sigma$ and it is regular because for all $t \geq 0$,

$$
\omega(f(\gamma(t)), f(\gamma(t+1))) \leq \omega(\gamma(t), \gamma(t+1)) .
$$

By Proposition 3.6, $f \circ \gamma$ converges to $\sigma$ non-tangentially. Corollary 3.11 implies then that $f\left(\gamma([0,+\infty)) \subset g(\mathbb{D})=\Delta\right.$. Hence, $\Delta \cap \Delta^{\prime} \neq \emptyset$, a contradiction.

Remark 4.19 By the previous proposition, if $\left(\mathbb{D}, g, \eta_{t}\right)$ and $\left(\mathbb{D}, \tilde{g}, \eta_{t}\right)$ are pre-models for $\left(\phi_{t}\right)$ at $\sigma$, then $g^{-1} \circ \tilde{g}$ is an automorphism of $\mathbb{D}$ which commutes with $\eta_{t}$ for all $t \geq 0$. Therefore, by a direct computation (or see [19]), $g^{-1} \circ \tilde{g}$ is a hyperbolic automorphism of $\mathbb{D}$ fixing $\pm \sigma$.

Given $z$ a point in a petal $\Delta$ of a semigroup $\left(\phi_{t}\right)$, the curve $\left.\mathbb{R} \ni t \mapsto \phi_{t}\right|_{\Delta}(z)$ is well defined. With a slight abuse of notation, we write $\phi_{t}(z)$ to denote $\left.\phi_{t}\right|_{\Delta}(z)$ for all $t \in \mathbb{R}$ when $z \in \Delta$.

Proposition 4.20 Let $\left(\phi_{t}\right)$ be a semigroup in $\mathbb{D}$ with a repelling fixed point $\sigma \in \partial \mathbb{D}$, with repelling spectral value $\lambda \in(-\infty, 0)$ and associated hyperbolic petal $\Delta$. Then, for all $z \in \Delta$,

$$
\lim _{t \rightarrow-\infty} \frac{1}{t} \log \left(1-\bar{\sigma} \phi_{t}(z)\right)=-\lambda,
$$

where $\log$ is the principal branch of the logarithm with $\operatorname{Im} \log (w) \in(-\pi, \pi]$ for all $w$. 
Proof Let $G$ be the infinitesimal generator of the semigroup. By [11],

$$
-\lambda=\angle \lim _{z \rightarrow \sigma} \frac{G(z)}{z-\sigma} \in(0, \infty) .
$$

Moreover, the regular backward orbit $[0,+\infty) \ni t \mapsto \phi_{-t}(z)$ converges to $\sigma$ nontangentially, so that

$$
-\lambda=\angle \lim _{t \rightarrow-\infty} \frac{G\left(\phi_{t}(z)\right)}{\phi_{t}(z)-\sigma}, \quad z \in \Delta .
$$

Given $z \in \Delta$ and $t \in \mathbb{R}$,

$$
\begin{aligned}
\int_{0}^{t} \frac{-\bar{\sigma} G\left(\phi_{s}(z)\right)}{1-\bar{\sigma} \phi_{s}(z)} \mathrm{d} s & =\int_{0}^{t} \frac{-\bar{\sigma}}{1-\bar{\sigma} \phi_{s}(z)} \frac{\partial \phi_{s}(z)}{\partial s} \mathrm{~d} s=\left[\log \left(1-\bar{\sigma} \phi_{s}(z)\right)\right]_{s=0}^{s=t} \\
& =\log \left(1-\bar{\sigma} \phi_{t}(z)\right)-\log (1-\bar{\sigma} z) .
\end{aligned}
$$

Then, using L'Hôpital's Rule and the non-tangential convergence, we obtain

$$
\lim _{t \rightarrow-\infty} \frac{1}{t} \int_{0}^{t} \frac{-\bar{\sigma} G\left(\phi_{s}\left(z_{0}\right)\right)}{1-\bar{\sigma} \phi_{s}\left(z_{0}\right)} \mathrm{d} s=\lim _{t \rightarrow+\infty} \frac{G\left(\phi_{t}\left(z_{0}\right)\right)}{\phi_{t}\left(z_{0}\right)-\sigma}=-\lambda .
$$

Hence, $\lim _{t \rightarrow-\infty} \frac{1}{t} \log \left(1-\bar{\sigma} \phi_{t}(z)\right)=-\lambda$.

As a last result of this section, we prove that a super-repelling fixed point can be the limit of at most one backward orbit:

Proposition 4.21 Let $\left(\phi_{t}\right)$ be a semigroup, not a group, in $\mathbb{D}$ with Denjoy-Wolff point $\tau \in \overline{\mathbb{D}}$. Suppose $\sigma \in \partial \mathbb{D}$ is a super-repelling fixed point of $\left(\phi_{t}\right)$. Assume $\gamma_{j}:[0,+\infty) \rightarrow \mathbb{D}, j=1,2$, are backward orbits of $\left(\phi_{t}\right)$ converging to $\sigma$. Then, either $\gamma_{1}([0,+\infty)) \subseteq \gamma_{2}([0,+\infty))$ or $\gamma_{2}([0,+\infty)) \subseteq \gamma_{1}([0,+\infty))$.

In particular, up to re-parameterization, there is at most one maximal invariant curve for $\left(\phi_{t}\right)$ with starting point $\sigma$.

Proof Suppose by contradiction that the statement is not true. Let define $\eta_{j}(t)=\gamma_{j}(-t)$ for $t \geq 0$ and $\eta_{j}(t)=\phi_{t}\left(\gamma_{j}(0)\right)$ for $t>0, j=1,2$. By Remark 4.4, $\eta_{1}, \eta_{2}$ are maximal invariant curves for $\left(\phi_{t}\right)$. Hence, by Remark 4.7, either $\eta_{1}((-\infty,+\infty))=\eta_{2}((-\infty,+\infty))$ or they are disjoint, and by our hypothesis, the latter case holds. Since $\lim _{t \rightarrow+\infty} \eta_{j}(t)=\tau$ and $\lim _{t \rightarrow-\infty} \eta_{j}(t)=\sigma, j=1,2$, by Remark 4.3 it follows that the closure of $\eta_{1}((-\infty,+\infty)) \cup$ $\eta_{2}((-\infty,+\infty))$, call it $J$, is a Jordan curve such that $J \cap \partial \mathbb{D}=\{\tau, \sigma\}$. Let $D$ be the bounded connected component of $\mathbb{C} \backslash J$. We claim that $D \subset \mathscr{W}$, the backward invariant set of $\left(\phi_{t}\right)$.

Assuming the claim, it follows at once that $D$ is contained in a petal $\Delta$. But $\sigma \in \partial \Delta$, hence, by Proposition 4.16, $\Delta$ is hyperbolic and $\sigma$ is repelling, which is a contradiction.

In order to prove the claim, let $z_{0} \in D$ and let $\eta:(a,+\infty) \rightarrow \mathbb{D}$ be the maximal invariant curve such that $\eta(0)=z_{0}$, with $a \in[-\infty, 0)$. Let $p \in \partial \mathbb{D}$ be the starting point of $\eta$. Since $\bar{D} \cap \partial \mathbb{D}=\{\sigma, \tau\}$, it follows that $p \in\{\tau, \sigma\}$; hence, Proposition 4.5 implies that $z_{0}$ belongs to the backward invariant set of $\left(\phi_{t}\right)$.

The last statement follows at once from what we already proved and Remark 4.4.

\section{Petals and the geometry of Koenigs functions}

In this section, we see how geometric properties of the Koenigs function of a semigroup detect petals. To achieve this goal, we use the divergence rate as introduced in [3], which, roughly 
speaking, measures the average hyperbolic speed of escape of an orbit of a semigroup. We recall from [3] the basic facts we need.

Definition 5.1 Let $\left(\phi_{t}\right)$ be a continuous one-parameter semigroup of holomorphic self-maps on a Riemann surface $\Omega$. Let $k_{\Omega}$ denote the hyperbolic distance of $\Omega$. Let $z \in \Omega$. The number

$$
c_{\Omega}\left(\phi_{t}\right):=\lim _{s \rightarrow+\infty} \frac{k_{\Omega}\left(\phi_{s}(z), z\right)}{s}
$$

exists independent of $z$ (see [3]) and is called the divergence rate of $\left(\phi_{t}\right)$.

Theorem 5.2 ([3]) Let $\left(\phi_{t}\right)$ be a non-elliptic holomorphic semigroup in $\mathbb{D}$ with Denjoy-Wolff point $\tau \in \partial \mathbb{D}$ and spectral value $\lambda \geq 0$. Let $c_{\mathbb{D}}\left(\phi_{t}\right)$ denote the divergence rate of $\left(\phi_{t}\right)$. Then,

$$
c_{\mathbb{D}}\left(\phi_{t}\right)=\frac{1}{2} \lambda \text {. }
$$

Moreover, if $\left(\Omega, h, \Phi_{t}\right)$ is a holomorphic model of $\left(\phi_{t}\right)$, then $c_{\mathbb{D}}\left(\phi_{t}\right)=c_{\Omega}\left(\Phi_{t}\right)$.

The basic observation we need is contained in the following lemma:

Lemma 5.3 Let $\left(\phi_{t}\right)$ be a semigroup, not a group, in $\mathbb{D}$. Let $\left(\Omega, h, \psi_{t}\right)$ be the canonical model of $\left(\phi_{t}\right)$ (where $\Omega$ and $\psi_{t}$ are given by Theorem 2.3). Suppose $\sigma \in \partial \mathbb{D}$ is a repelling fixed point for $\left(\phi_{t}\right)$ with repelling spectral value $\lambda \in(-\infty, 0)$. Let $\Delta$ be the hyperbolic petal of $\left(\phi_{t}\right)$ such that $\sigma \in \partial \Delta$. Let $A:=h(\Delta)$. Then, $\psi_{t}(A)=A$ for all $t \geq 0$ and $\left(\left.\psi_{t}\right|_{A}\right)$ is a continuous group of automorphisms of A. Moreover, the divergence rate satisfies

$$
c_{\Delta}\left(\left.\phi_{t}\right|_{\Delta}\right)=c_{A}\left(\left.\psi_{t}\right|_{A}\right)=-\frac{\lambda}{2} .
$$

Proof Let $\left(\mathbb{D}, g, \eta_{t}\right)$ be a pre-model for $\left(\phi_{t}\right)$ at $\sigma$. By Proposition $4.18, g(\mathbb{D})=\Delta$. Hence, $\left(\Delta, g,\left.\phi_{t}\right|_{\Delta}\right)$ is a holomorphic model for $\left(\eta_{t}\right)$. Therefore, by Theorem 5.2,

$$
c_{\Delta}\left(\left.\phi_{t}\right|_{\Delta}\right)=c_{\mathbb{D}}\left(\eta_{t}\right)=-\frac{\lambda}{2} .
$$

Now, let $A=h(\Delta)$. From

$$
A=h(\Delta)=h\left(\phi_{t}(\Delta)\right)=\psi_{t}(h(\Delta))=\psi_{t}(A), \quad t \geq 0,
$$

it follows that $\left(\left.\psi_{t}\right|_{A}\right)$ is a continuous group of automorphisms of $A$ and that $\left(\Delta,\left.h\right|_{\Delta},\left(\left.\phi_{t}\right|_{\Delta}\right)\right)$ is a holomorphic model for $\left(\left.\psi_{t}\right|_{A}\right)$. Hence, again by Theorem 5.2 and (5.2), we have the result.

Now, we need to introduce spirallike sectors.

Definition 5.4 Let $\mu \in \mathbb{C}$, $\operatorname{Re} \mu>0, \alpha \in(0, \pi]$ and $\theta_{0} \in[-\pi, \pi)$. A $\mu$-spirallike sector of amplitude $2 \alpha$ and center $e^{i \theta_{0}}$ is

$$
\operatorname{Spir}\left[\mu, 2 \alpha, \theta_{0}\right]:=\left\{e^{t \mu+i \theta}: t \in \mathbb{R}, \theta \in\left(-\alpha+\theta_{0}, \alpha+\theta_{0}\right)\right\} .
$$

Lemma 5.5 Let $D:=\operatorname{Spir}\left[\mu, 2 \alpha, \theta_{0}\right]$ be a $\mu$-spirallike sector for some $\mu \in \mathbb{C}, \operatorname{Re} \mu>0$ and $\alpha \in(0, \pi]$. Let $\psi_{t}(z)=e^{-\mu t} z$, for $z \in \mathbb{C}$. Then, $\left(\left.\psi_{t}\right|_{D}\right)$ is a continuous group of automorphisms of $D$ and

$$
c_{D}\left(\left.\psi_{t}\right|_{D}\right)=\frac{|\mu|^{2} \pi}{4 \alpha \operatorname{Re} \mu} .
$$


Proof The map $z \mapsto e^{-i \theta_{0}} z$ is a biholomorphism between $D$ and $\operatorname{Spir}[\mu, 2 \alpha, 0]$ and conjugate $\left(\left.\psi_{t}\right|_{D}\right)$ to $\left(\left.\psi_{t}\right|_{\operatorname{Spir}[\mu, 2 \alpha, 0]}\right)$. Since a biholomorphism is an isometry with respect to the hyperbolic distance, the divergence rate of $\left(\left.\psi_{t}\right|_{D}\right)$ and $\left(\left.\psi_{t}\right|_{\operatorname{Spir}[\mu, 2 \alpha, 0]}\right)$ is the same. Hence, we can assume $\theta_{0}=0$.

It is clear that $\psi_{t}(D)=D$ for all $t \geq 0$; hence, $\left(\left.\psi_{t}\right|_{D}\right)$ is a continuous group of automorphisms of $D$. Since $D$ is simply connected and $0 \notin D$, a holomorphic branch of $f: D \ni w \mapsto w^{1-i \frac{\operatorname{lm} \mu}{\operatorname{Re} \mu}} \in \mathbb{C}$ is well defined. A straightforward computation shows that $D^{\prime}:=f(D)=\left\{\rho e^{i \theta}: \rho>0, \theta \in(-\alpha, \alpha)\right\}$.

Moreover, $\left(f \circ \psi_{t} \circ f^{-1}\right)$ is a continuous group of automorphisms of $D^{\prime}$. A direct computation shows

$$
\tilde{\psi}_{t}(z):=\left(f \circ \psi_{t} \circ f^{-1}\right)(z)=e^{-\mu t\left(1-i \frac{\operatorname{lm} \mu}{\operatorname{Re} \mu}\right)} z=e^{-t \frac{|\mu|^{2}}{\operatorname{Re} \mu}} z,
$$

for all $t \geq 0$. Now, consider the function $g: D^{\prime} \ni z \mapsto z^{-\frac{\pi}{2 \alpha}} \in \mathbb{C}$. Then, $g\left(D^{\prime}\right)=\mathbb{H}$ and $\left(g \circ \tilde{\psi}_{t} \circ g^{-1}\right)$ is a continuous group of automorphisms of $\mathbb{H}$. A direct computation shows that $\eta_{t}(z):=\left(g \circ \tilde{\psi}_{t} \circ g^{-1}\right)(z)=e^{t \frac{\pi|\mu|^{2}}{2 \alpha \operatorname{Re} \mu}} z$ for all $t \geq 0$. That is, $\left(\eta_{t}\right)$ is a group in $\mathbb{H}$ which is conjugated to a hyperbolic group $\left(\tilde{\eta}_{t}\right)$ in $\mathbb{D}$ with spectral value $\frac{\pi|\mu|^{2}}{2 \alpha \operatorname{Re} \mu}$ and then

$$
c_{\mathbb{D}}\left(\tilde{\eta}_{t}\right)=\frac{\pi|\mu|^{2}}{4 \alpha \operatorname{Re} \mu} .
$$

Since $\left(D, g \circ f,\left(\left.\psi_{t}\right|_{D}\right)\right)$ is a holomorphic model for $\left(\tilde{\eta}_{t}\right)$, it follows $c\left(\tilde{\eta}_{t}\right)=c_{D}\left(\left.\psi_{t}\right|_{D}\right)$ by Theorem 5.2.

We are now ready to relate petals of a semigroup with the shape of the image of the corresponding Koenigs function. As a matter of notation, if $D \subset \mathbb{C}$ is a $\mu$-starlike domain with respect to 0 for some $\mu \in \mathbb{C}$, $\operatorname{Re} \mu>0$, we say that a $\mu$-spirallike sector $\operatorname{Spir}\left[\mu, 2 \alpha, \theta_{0}\right] \subset D$ (for some $\alpha \in[0, \pi)$ and $\theta_{0} \in[-\pi, \pi)$ ) is maximal if there exist no $\theta_{1} \in[-\pi, \pi), \beta \in(0,2 \pi]$ such that $\operatorname{Spir}\left[\mu, 2 \alpha, \theta_{0}\right] \subset \operatorname{Spir}\left[\mu, \beta, \theta_{1}\right] \subset D$ and $\operatorname{Spir}\left[\mu, 2 \alpha, \theta_{0}\right] \neq \operatorname{Spir}\left[\mu, \beta, \theta_{1}\right]$.

Similarly, if $D \subset \mathbb{C}$ is starlike at infinity, $z_{0} \in \mathbb{C}, \rho>0$, the strip $\left(\mathbb{S}_{\rho}+z_{0}\right) \subset D$ is maximal if there exist no $r>0$ and $z_{1} \in \mathbb{C}$ such that $\left(\mathbb{S}_{\rho}+z_{0}\right) \subset\left(\mathbb{S}_{r}+z_{1}\right) \subset D$ and $\left(\mathbb{S}_{\rho}+z_{0}\right) \neq\left(\mathbb{S}_{r}+z_{1}\right)$.

In the discrete (elliptic, starlike type) case, Theorem 5.6 was proven in [20]. For semigroups, Theorems 5.6 and 5.7 were first proved with a direct, lengthy and more complicated argument in [9].

Theorem 5.6 Let $\left(\phi_{t}\right)$ be a semigroup, not a group, in $\mathbb{D}$. Let $\tau \in \overline{\mathbb{D}}$ be the Denjoy-Wolff point of $\left(\phi_{t}\right)$ and $\mu$ its spectral value. Let $h$ be the Koenigs function of $\left(\phi_{t}\right)$. Suppose $\Delta$ is a hyperbolic petal for $\left(\phi_{t}\right)$, let $\sigma \in \partial \Delta$ be the unique repelling fixed point for $\left(\phi_{t}\right)$ on $\partial \mathbb{D}$, and let $\lambda \in(-\infty, 0)$ be the repelling spectral value of $\left(\phi_{t}\right)$ at $\sigma$.

(1) If $\tau \in \mathbb{D}$, then there exists $\theta_{0} \in[-\pi, \pi)$ such that $h(\Delta)$ is a maximal spirallike sector of $h(\mathbb{D})$ of center $e^{i \theta_{0}}$ and amplitude $-\frac{|\mu|^{2} \pi}{\lambda \operatorname{Re} \mu}$, i.e.,

$$
h(\Delta)=\operatorname{Spir}\left[\mu,-\frac{|\mu|^{2} \pi}{\lambda \operatorname{Re} \mu}, \theta_{0}\right] .
$$

(2) If $\tau \in \partial \mathbb{D}$, then there exists $z_{0} \in \mathbb{C}$ such that $h(\Delta)$ is a maximal strip $z_{0}+\mathbb{S}_{-\frac{\pi}{\lambda}}$, i.e.,

$$
h(\Delta)=z_{0}+\mathbb{S}_{-\frac{\pi}{\lambda}} .
$$


Proof We first consider the elliptic case. The canonical model is $\left(\mathbb{C}, h, e^{-\mu t} z\right)$. Since $\phi_{t}(\Delta)=$ $\Delta$ for all $t \geq 0$, it follows that $e^{-\mu t} h(\Delta)=h(\Delta)$ and $z \mapsto e^{-\mu t} z$ is an automorphism of $h(\Delta)$ for all $t \geq 0$. In particular, $e^{-\mu t} h(\Delta)=h(\Delta)$ for all $t \in \mathbb{R}$. It follows easily that $h(\Delta)=\operatorname{Spir}\left[\mu, 2 \alpha, \theta_{0}\right]$ for some $\theta_{0} \in[-\pi, \pi)$ and $\alpha \in(0, \pi]$. By Lemmas 5.3 and 5.5 it follows at once that $2 \alpha=-\frac{|\mu|^{2} \pi}{\lambda \operatorname{Re} \mu}$.

It is left to prove that $\operatorname{Spir}\left[\mu,-\frac{|\mu|^{2} \pi}{\lambda \operatorname{Re} \mu}, \theta_{0}\right]$ is maximal. Suppose this is not the case. Therefore, there exist $\theta_{1} \in[-\pi, \pi), \beta \in(0,2 \pi]$ such that $D:=\operatorname{Spir}\left[\mu, \beta, \theta_{1}\right] \subset h(\mathbb{D})$ and $\operatorname{Spir}\left[\mu,-\frac{|\mu|^{2} \pi}{\lambda \operatorname{Re} \mu}, \theta_{0}\right]$ is properly contained in $D$. Therefore, $\left(\left.e^{-\mu t} z\right|_{D}\right)$ is a continuous group of automorphisms of $D$. Since $D \subset h(\mathbb{D})$, it follows $\phi_{t}\left(h^{-1}(z)\right)=h^{-1}\left(e^{-\mu t} z\right)$ for every $z \in h(\mathbb{D})$; hence $\phi_{t}\left(h^{-1}(D)\right)=h^{-1}(D)$ for all $t \geq 0$. This implies that $h^{-1}(D)$ is an open connected component in the backward invariant set of $\left(\phi_{t}\right)$ which properly contains $\Delta$, a contradiction.

The proof in case $\left(\phi_{t}\right)$ is non-elliptic is similar, and we leave the details to the reader.

The converse of the previous theorem is also true:

Theorem 5.7 Let $\left(\phi_{t}\right)$ be a semigroup, not a group, in $\mathbb{D}$. Let $\tau \in \overline{\mathbb{D}}$ be the Denjoy-Wolff point of $\left(\phi_{t}\right)$ and $\mu$ its spectral value. Let $h$ be the Koenigs function of $\left(\phi_{t}\right)$.

(1) If $\tau \in \mathbb{D}$ and there exist $\theta_{0} \in[-\pi, \pi)$ and $\beta \in(0,2 \pi]$ such that $\operatorname{Spir}\left[\mu, \beta, \theta_{0}\right] \subset h(\mathbb{D})$ is a maximal spirallike sector of $h(\mathbb{D})$, then $h^{-1}\left(\operatorname{Spir}\left[\mu, \beta, \theta_{0}\right]\right)$ is a hyperbolic petal for $\left(\phi_{t}\right)$. Moreover, if $\sigma \in \partial \Delta$ is the unique repelling fixed point of $\left(\phi_{t}\right)$ contained in $\partial \Delta$, then the repelling spectral value of $\left(\phi_{t}\right)$ at $\sigma$ is $\lambda=-\frac{|\mu|^{2} \pi}{\beta \operatorname{Re} \mu}$.

(2) If $\tau \in \partial \mathbb{D}$ and there exist $z_{0} \in \mathbb{C}$ and $\rho>0$ such that $z_{0}+\mathbb{S}_{\rho} \subset h(\mathbb{D})$ is a maximal strip of $h(\mathbb{D})$, then $h^{-1}\left(z_{0}+\mathbb{S}_{\rho}\right)$ is a hyperbolic petal for $\left(\phi_{t}\right)$. Moreover, if $\sigma \in \partial \Delta$ is the unique repelling fixed point of $\left(\phi_{t}\right)$ contained in $\partial \Delta$, then the repelling spectral value of $\left(\phi_{t}\right)$ at $\sigma$ is $\lambda=-\frac{\pi}{\rho}$.

Proof (1) The canonical model is $\left(\mathbb{C}, h, e^{-\mu t} z\right)$. Let $D:=\operatorname{Spir}\left[\mu, \beta, \theta_{0}\right]$. Since $\phi_{t}\left(h^{-1}(z)=\right.$ $h^{-1}\left(e^{-\mu t} z\right)$ for all $z \in h(\mathbb{D})$ and $t \geq 0$, it follows at once that $\phi_{t}(D)=D$ for all $t \geq 0$. Hence, there exists a petal $\Delta$ such that $h^{-1}(D) \subseteq \Delta$. However, if $\Delta \neq h^{-1}(D)$, then by Theorem $5.6, h(\Delta)$ is a spirallike sector properly containing $D$, against the maximality of $D$. Therefore, $\Delta=h^{-1}(D)$ and the result follows from Theorem 5.6.

(2) The argument is similar and we omit it.

Finally, we turn our attention to parabolic petals. Recall that only parabolic semigroups can have parabolic petals (Remark 4.11).

As a matter of notation, if $W \subset \mathbb{C}$ is a domain starlike at infinity and $a \in \mathbb{R}$, we say that a half-plane $\{w \in \mathbb{C}: \operatorname{Re} w>a\} \subset W$ (respectively $\{w \in \mathbb{C}: \operatorname{Re} w<a\} \subset W$ ) is maximal if $\{w \in \mathbb{C}: \operatorname{Re} w>b\} \not \subset W$ for every $b<a$ (respect. $\{w \in \mathbb{C}: \operatorname{Re} w<b\} \not \subset W$ for every $b>a)$.

Theorem 5.8 Let $\left(\phi_{t}\right)$ be a parabolic semigroup, not a group, in $\mathbb{D}$, with Denjoy-Wolff point $\tau \in \partial \mathbb{D}$. Let $h$ be the Koenigs function of $\left(\phi_{t}\right)$. If $\Delta$ is a parabolic petal for $\left(\phi_{t}\right)$, then $h(\Delta)$ is a maximal half-plane in $h(\mathbb{D})$. Conversely, if $H \subset h(\mathbb{D})$ is a maximal half-plane in $h(\mathbb{D})$ then $h^{-1}(H)$ is a parabolic petal for $\left(\phi_{t}\right)$.

Moreover, $\left(\phi_{t}\right)$ can have at most two parabolic petals and, if this is the case, $\left(\phi_{t}\right)$ has zero hyperbolic step. 
Proof Let $(\Omega, h, z+i t)$ be the canonical model of $\left(\phi_{t}\right)$, where $\Omega=\mathbb{C}, \mathbb{H}$ or $\mathbb{H}^{-}$. Let $\Delta$ be a parabolic petal for $\left(\phi_{t}\right)$. Since $\phi_{t}(\Delta)=\Delta$ for all $t \geq 0$, and $h(\Delta)=h\left(\phi_{t}(\Delta)\right)=h(\Delta)+i t$ for all $t \geq 0$, it follows that $h(\Delta)+i t=h(\Delta)$ for all $t \geq 0$, and, hence, $h(\Delta)+i t=h(\Delta)$ for all $t \in \mathbb{R}$. Therefore, either $h(\Delta)$ is a strip $\mathbb{S}_{\rho}+z_{0}$ for some $\rho>0$ and $z_{0} \in h(\mathbb{D})$ or $h(\Delta)$ is a half-plane. Arguing as in the proof of Theorem 5.6, it is easy to see that $h(\Delta)$ is maximal in $h(\mathbb{D})$. Therefore, if $h(\Delta)$ is a maximal strip, the petal $\Delta$ is hyperbolic by Theorem 5.7, contradicting our hypothesis. Hence, $h(\Delta)$ is a maximal half-plane in $h(\mathbb{D})$.

Conversely, if $H \subset h(\mathbb{D})$ is a maximal half-plane in $h(\mathbb{D})$, then arguing as in the proof of Theorem 5.7, it follows that $h^{-1}(H)$ is a petal. Moreover, by Theorem 5.6, $h^{-1}(H)$ cannot be hyperbolic; hence, it is parabolic.

It is clear that a domain starlike at infinity (different from $\mathbb{C}$ ) can contain at most two maximal half-planes, one given by $\{w \in \mathbb{C}: \operatorname{Re} w<a\}$ and the other given by $\{w \in \mathbb{C}$ : Re $w>b$, for some $-\infty<a \leq b<+\infty$. Hence, $\left(\phi_{t}\right)$ can have at most two parabolic petals.

Finally, assume that $\left(\phi_{t}\right)$ has two parabolic petals $\Delta_{1}, \Delta_{2}$. Hence, there exist $-\infty<a \leq$ $b<+\infty$ such that $h\left(\Delta_{1}\right)=\{w \in \mathbb{C}: \operatorname{Re} w<a\}$ and $h\left(\Delta_{2}\right)=\{w \in \mathbb{C}: \operatorname{Re} w>b\}$. This implies that $h(\mathbb{D})$ is not contained in $\mathbb{H}$ or $\mathbb{H}^{-}$, and by Theorem 2.3, it follows that $\Omega=\mathbb{C}$ and $\left(\phi_{t}\right)$ has zero hyperbolic step.

\section{Analytic properties of Koenigs functions at boundary fixed points}

We start by recalling the following straightforward consequence of [18, Prop. 3.4, Prop. 3.7]:

Proposition 6.1 Let $\left(\phi_{t}\right)$ be a semigroup, not a group, in $\mathbb{D}$ with Denjoy-Wolff point $\tau \in \overline{\mathbb{D}}$ and Koenigs function $h$. Then, $\sigma \in \partial \mathbb{D}$ is a fixed point of $\left(\phi_{t}\right)$ if and only if $\angle \lim _{z \rightarrow \sigma} h(z)=$ $\infty \in \mathbb{C}_{\infty}$. Moreover, if $\sigma \neq \tau$, then (the unrestricted limit) $\lim _{z \rightarrow \sigma} h(z)=\infty$.

The aim of this section is to characterize repelling and super-repelling fixed points via Koenigs functions. In order to properly deal with the elliptic case, we need to introduce some terminology.

Let $\lambda \in \mathbb{C}, \operatorname{Re} \lambda>0$. Every point $w \in \mathbb{C} \backslash\{0\}$ can be written in a unique way in $\lambda$-spirallike coordinates as $w=e^{-\lambda t+i \theta}$, where $t \in \mathbb{R}$ and $\theta \in[-\pi, \pi)$. We define

$$
\operatorname{Arg}_{\lambda}(w):=\theta,
$$

and we call it the $\lambda$-spirallike argument of $w$.

Theorem 6.2 Let $\left(\phi_{t}\right)$ be an elliptic semigroup in $\mathbb{D}$, not a group, with Denjoy-Wolff point $\tau \in \mathbb{D}$ and spectral value $\lambda \in \mathbb{C}$ with $\operatorname{Re} \lambda>0$. Let $h$ be the associated Koenigs function and $\sigma \in \partial \mathbb{D}$. The following are equivalent:

(1) $\sigma$ is a repelling fixed point of $\left(\phi_{t}\right)$,

(2) $\lim _{z \rightarrow \sigma}|h(z)|=\infty$ and $\angle \liminf _{z \rightarrow \sigma} \operatorname{Arg}_{\lambda}(h(z)) \neq \angle \limsup _{z \rightarrow \sigma} \operatorname{Arg}_{\lambda}(h(z))$,

(3) $\lim _{z \rightarrow \sigma}|h(z)|=\infty$ and $\liminf _{z \rightarrow \sigma} \operatorname{Arg}_{\lambda}(h(z)) \neq \limsup _{z \rightarrow \sigma} \operatorname{Arg}_{\lambda}(h(z))$.

Moreover, if $\sigma$ is a repelling fixed point for $\left(\phi_{t}\right)$ with repelling spectral value $v \in(-\infty, 0)$, then there exists $\theta_{0} \in[-\pi, \pi)$ such that if $\left\{z_{n}\right\} \subset \mathbb{D}$ is a sequence converging to $\sigma$ and $\lim _{n \rightarrow \infty} \operatorname{Arg}\left(1-\bar{\sigma} z_{n}\right)=\beta \in(-\pi / 2, \pi / 2)$, then

$$
\lim _{n \rightarrow \infty} \operatorname{Arg}_{\lambda}\left(h\left(z_{n}\right)\right)=\theta_{0}+\frac{\beta|\lambda|^{2}}{v \operatorname{Re} \lambda} \bmod [-\pi, \pi) .
$$


Proof (1) implies (2). Suppose $\sigma$ is a repelling fixed point for $\left(\phi_{t}\right)$ with spectral value $v \in$ $(-\infty, 0)$. By Proposition 6.1, $\lim _{z \rightarrow \sigma}|h(z)|=\infty$. By Theorem 5.6, there exists a hyperbolic petal $\Delta$ such that $\sigma \in \partial \Delta$ and $h(\Delta)=\operatorname{Spir}\left[\lambda, 2 \alpha, \theta_{0}\right]$ is a maximal $\lambda$-spirallike sector of amplitude $2 \alpha$ and center $e^{i \theta_{0}}$ in $h(\mathbb{D})$, where $\theta_{0} \in[0,2 \pi)$ and $2 \alpha=-\pi|\lambda|^{2} / \nu \operatorname{Re} \lambda$. Let $\left(\mathbb{D}, g, \eta_{t}\right)$ be a pre-model for $\left(\phi_{t}\right)$ at $\sigma$. By Proposition 4.18, $g(\mathbb{D})=\Delta$. Now, take a holomorphic branch of $f: z \mapsto z^{1-i \frac{\operatorname{lm} \lambda}{\operatorname{Re} \lambda}}$ such that the image of $\operatorname{Spir}\left[\lambda, 2 \alpha, \theta_{0}\right]$ is $V=$ $\left\{\rho e^{i \theta}: \rho>0, \theta \in\left(\theta_{0}-\alpha, \theta_{0}+\alpha\right)\right\}$. Let then $k: V \rightarrow \mathbb{H}$ be defined by $k(w)=w^{-\pi / 2 \alpha}$. By construction, $C:=k \circ f \circ h \circ g: \mathbb{D} \rightarrow \mathbb{H}$ is a biholomorphism. Hence, $C$ is a Möbius transformation, and, looking at the definition and taking into account that $\lim _{z \rightarrow \sigma}|h(z)|=\infty$, we see that $C(\sigma)=0$ and $C(-\sigma)=\infty$. Therefore,

$$
C(z)=\frac{\sigma-z}{\sigma+z}
$$

Now, let $\left\{z_{n}\right\} \subset \mathbb{D}$ be a sequence converging to $\sigma$ such that $\lim _{n \rightarrow \infty} \operatorname{Arg}\left(1-\bar{\sigma} z_{n}\right)=\beta \in$ $(-\pi / 2, \pi / 2)$. By Proposition $4.18,\left\{z_{n}\right\}$ is eventually contained in $g(\mathbb{D})$ and, without loss of generality, we can assume $\left\{z_{n}\right\} \subset g(\mathbb{D})$. Let $w_{n}:=g^{-1}\left(z_{n}\right)$. Since $\angle \lim _{z \rightarrow \sigma} g(z)=\sigma$ and $g$ is semi-conformal at $\sigma$, it follows at once that $\lim _{n \rightarrow \infty} \operatorname{Arg}\left(1-\bar{\sigma} w_{n}\right)=\beta$. Hence, $\lim _{n \rightarrow \infty} \operatorname{Arg}\left(C\left(w_{n}\right)\right)=\beta$. Therefore, taking into account that $\operatorname{Arg}_{\lambda} f^{-1}(w)=\operatorname{Arg}(w)$ for all $w \in V$, we have

$$
\lim _{n \rightarrow \infty} \operatorname{Arg}_{\lambda}\left(h\left(z_{n}\right)\right)=\lim _{n \rightarrow \infty} \operatorname{Arg}_{\lambda}\left(f^{-1} \circ k^{-1} \circ C \circ g^{-1}\right)\left(z_{n}\right)=\lim _{n \rightarrow \infty} \operatorname{Arg}\left(k^{-1} \circ C\right)\left(w_{n}\right) .
$$

Taking into account that $k^{-1}(z)=e^{i \theta_{0}} z^{-2 \alpha / \pi}$, the previous equation gives immediately

$$
\lim _{n \rightarrow \infty} \operatorname{Arg}_{\lambda}\left(h\left(z_{n}\right)\right)=\theta_{0}-\frac{2 \alpha \beta}{\pi} \bmod [-\pi, \pi) .
$$

This proves (2) and, since $2 \alpha=-\pi|\lambda|^{2} / \nu \operatorname{Re} \lambda$, the final part of the statement.

Clearly, (2) implies (3).

(3) implies (1). Since $\lim _{z \rightarrow \sigma}|h(z)|=\infty$ implies $\lim _{z \rightarrow \sigma} h(z)=\infty$ in $\mathbb{C}_{\infty}$, Proposition 6.1 immediately implies that $\sigma$ is a boundary fixed point of $\left(\phi_{t}\right)$. We have to show that $\sigma$ is repelling. Let $\underline{x}_{\sigma} \in \partial_{C} \mathbb{D}$ be the prime end representing $\sigma$ and let $\hat{h}: \widehat{\mathbb{D}} \rightarrow \widehat{h(\mathbb{D})}$ be the homeomorphism in the Carathéodory topology defined by $h$. Since $\lim _{z \rightarrow \sigma} h(z)=\infty$, we have $I\left(\hat{h}\left(\underline{x}_{\sigma}\right)\right)=\{\infty\}$, where $I\left(\hat{h}\left(\underline{x}_{\sigma}\right)\right)$ is the impression of $\hat{h}\left(\underline{x}_{\sigma}\right)$. Hence, we can find a circular null chain $\left(C_{n}\right)$ representing $\hat{h}\left(\underline{x}_{\sigma}\right)$ such that there exists an increasing sequence of positive real numbers $\left\{R_{n}\right\}$ converging to $+\infty$ such that $C_{n} \subset\left\{z \in \mathbb{C}:|z|=R_{n}\right\}$ for every $n \in \mathbb{N}_{0}$. For every $n \in \mathbb{N}_{0}$, let $e^{-\lambda t_{n}+i \theta_{n}^{1}}$ and $e^{-\lambda t_{n}+i \theta_{n}^{2}}$ be the end points of $\overline{C_{n}}$, where $t_{n} \in \mathbb{R}$ is such that $e^{-t_{n} \operatorname{Re} \lambda}=R_{n}$ and $\theta_{n}^{j} \in[-\pi, \pi) j=1,2$ with $\theta_{n}^{1} \leq \theta_{n}^{2}$.

Given $\mu \in \mathbb{C}$ with $\operatorname{Re} \mu>0$ and $c \in \mathbb{C} \backslash\{0\}$, we let

$$
\operatorname{spir}_{\mu}[c]:=\left\{e^{-\mu s} c: s \in \mathbb{R}\right\} \cup\{0\}
$$

If $\theta_{n}^{1}=\theta_{n}^{2}$ for all $n \in \mathbb{N}$, then $C_{n}=\left\{z \in \mathbb{C}:|z|=R_{n}\right\} \backslash\left\{e^{-\lambda t_{n}+i \theta_{n}^{1}}\right\}$ for all $n \in \mathbb{N}$. Since $h(\mathbb{D})$ is $\lambda$-spirallike, it follows that for all $n \in \mathbb{N}$,

$$
h(\mathbb{D}) \cap\left(\operatorname{spir}_{\lambda}\left[e^{-\lambda t_{n}+i \theta_{n}^{1}}\right] \cap\left\{w \in \mathbb{C}:|w| \geq R_{n}\right\}\right)=\emptyset .
$$

Hence, the only possibility is that $\theta_{n}^{1}$ is constant for all $n$, say, $\theta_{n}^{1}=: \theta_{0}$. That is, $h(\mathbb{D})=$ $\mathbb{C} \backslash\left(\operatorname{spir}_{\lambda}\left[e^{i \theta_{0}}\right] \cap\{w \in \mathbb{C}:|w| \geq R\}\right)$, for some $R \in\left(0, R_{0}\right]$. It is then clear that $\mathbb{C} \backslash \operatorname{spir}_{\lambda}\left[e^{i \theta_{0}}\right]$ is a maximal $\lambda$-spirallike sector in $h(\mathbb{D})$ of amplitude $2 \pi$. By Theorem 5.7, there exists a hyperbolic petal $\Delta \subset \mathbb{D}$ such that $h(\Delta)=\mathbb{C} \backslash \operatorname{spir}_{\lambda}\left[e^{i \theta_{0}}\right]$. Moreover, if $J:=\left(\operatorname{spir}_{\lambda}\left[e^{i \theta_{0}}\right] \cap\right.$ 
$\{w \in \mathbb{C}:|w|<R\})$, then $\Delta=\mathbb{D} \backslash h^{-1}(J)$. Note that, by [26, Lemma 2, p. 162], the closure of $h^{-1}(J)$ is a Jordan arc with end points $\tau \in \mathbb{D}$ and a point $p \in \partial \mathbb{D}$. Hence, $\partial \Delta=h^{-1}(J) \cup \partial \mathbb{D}$. By Proposition 4.16, $\partial \Delta$ contains only one boundary fixed point of $\left(\phi_{t}\right)$, which is repelling. Since $\sigma \in \partial \Delta$ is a fixed point, it follows that $\sigma$ is repelling.

Now, we assume that there exists $n_{0} \in \mathbb{N}$ such that $\theta_{n_{0}}^{1}<\theta_{n_{0}}^{2}$. Up to considering the equivalent null chain $\left(C_{n}\right)_{n \geq n_{0}}$, we can assume $n_{0}=0$. Since $h(\mathbb{D})$ is $\lambda$-spirallike, $h(\mathbb{D}) \cap$ $\left(\operatorname{spir}_{\lambda}\left[e^{i \theta_{0}^{j}}\right] \cap\left\{w \in \mathbb{C}:|w| \geq R_{0}\right\}\right)=\emptyset, j=1,2$. Hence, it is easy to see that $\theta_{n}^{1}<\theta_{n}^{2}$ for all $n \geq 0$. Let $V_{n}$ be the interior part of $C_{n}, n \geq 1$. Since $\operatorname{spir}_{\lambda}\left[e^{i \theta_{0}^{1}}\right] \cup \operatorname{spir}_{\lambda}\left[e^{i \theta_{0}^{2}}\right] \cup\{\infty\}$, forms a Jordan curve $J$ in the Riemann sphere $\mathbb{C}_{\infty}$ containing 0 and $\infty$, taking into account that $h(\mathbb{D})$ is $\lambda$-spirallike, it follows that $V_{n}$ is contained in one of the connected components of $\mathbb{C} \backslash J$. Thus, taking also into account that for every $w \in C_{n}$, we have $\left(\operatorname{spir}_{\lambda}[w] \cap\{w \in \mathbb{C}\right.$ : $\left.\left.|w|<R_{n}\right\}\right) \subset h(\mathbb{D})$, we have two possibilities. Either

$$
\begin{gathered}
V_{n} \subseteq\left(\operatorname{Spir}\left[\lambda, \theta_{n}^{2}-\theta_{n}^{1}, \frac{\theta_{n}^{2}+\theta_{n}^{1}}{2}\right] \cap\left\{w \in \mathbb{C}:|w|>R_{n}\right\}\right), \\
\left(\operatorname{Spir}\left[\lambda, \theta_{n}^{2}-\theta_{n}^{1}, \frac{\theta_{n}^{2}+\theta_{n}^{1}}{2}\right] \cap\left\{w \in \mathbb{C}:|w|<R_{n}\right\}\right) \subset h(\mathbb{D})
\end{gathered}
$$

or, setting $\xi_{n}:=\frac{\theta_{n}^{2}+\theta_{n}^{1}+2 \pi}{2} \bmod 2 \pi$,

$$
\begin{aligned}
& V_{n} \subseteq\left(\operatorname{Spir}\left[\lambda, \theta_{n}^{1}-\theta_{n}^{2}+2 \pi, \xi_{n}\right] \cap\left\{w \in \mathbb{C}:|w|>R_{n}\right\}\right), \\
& \quad\left(\operatorname{Spir}\left[\lambda, \theta_{n}^{1}-\theta_{n}^{2}+2 \pi, \xi_{n}\right] \cap\left\{w \in \mathbb{C}:|w|<R_{n}\right\}\right) \subset h(\mathbb{D}) .
\end{aligned}
$$

If (6.2) holds for some $n=n_{0}$, since $V_{n} \subset V_{n_{0}}$ for all $n \geq n_{0}$, (6.2) implies that $\theta_{n}^{1}<\theta_{n}^{2}$ and (6.2) holds for all $n \geq n_{0}$. Assume we are in this case-the proof for the case (6.3) holds for every $n$ is similar and we omit it.

Again, we can assume $n_{0}=0$. By hypothesis, there exist two sequences $\left\{z_{m}\right\},\left\{w_{m}\right\} \subset \mathbb{D}$ converging to $\sigma$ such that $\alpha:=\lim _{m \rightarrow \infty} \operatorname{Arg}_{\lambda}\left(h\left(z_{m}\right)\right)$ and $\beta:=\lim _{m \rightarrow \infty} \operatorname{Arg}_{\lambda}\left(h\left(w_{m}\right)\right)$ exist and $\alpha<\beta$. Let us write $h\left(z_{m}\right)=e^{-\lambda r_{m}+i \alpha_{m}}$ and $h\left(w_{m}\right)=e^{-\lambda s_{m}+i \beta_{m}}$, where $r_{m}, s_{m} \in \mathbb{R}$ and $\alpha_{m}, \beta_{m} \in[-\pi, \pi)$ have the property that $\lim _{m \rightarrow \infty} \alpha_{m}=\alpha$ and $\lim _{m \rightarrow \infty} \beta_{m}=\beta$. Since $\left\{z_{m}\right\}$ and $\left\{w_{m}\right\}$ are converging to $\sigma$ in the Euclidean topology, it follows that they also converge to $\underline{x}_{\sigma}$ in the Carathéodory topology of $\mathbb{D}$. Hence, $\left\{h\left(z_{m}\right)\right\}$ and $\left\{h\left(w_{m}\right)\right\}$ converge to $\hat{h}\left(\underline{x}_{\sigma}\right)$ in the Carathéodory topology of $h(\mathbb{D})$. It follows that for all $n \in \mathbb{N}$ there exists $m_{n} \in \mathbb{N}$ such that $h\left(z_{m}\right), h\left(w_{m}\right) \in V_{n}$ for all $m \geq m_{n}$. By (6.2), we have $\theta_{n}^{1}<\alpha_{m}, \beta_{m}<\theta_{n}^{2}$ for all $n$ and $m \geq m_{n}$, and, taking the limit in $m$, we obtain

$$
\theta_{n}^{1} \leq \alpha<\beta \leq \theta_{n}^{2}, \quad n \in \mathbb{N} .
$$

Let $\theta_{0}:=(\beta+\alpha) / 2$ and $a \in(0, \beta-\alpha)$. Equation in (6.4) implies at once that $\operatorname{Spir}\left[\lambda, a, \theta_{0}\right] \cap$ $\left\{w \in \mathbb{C}:|w|>R_{n}\right\} \subset V_{n}$ for all $n \in \mathbb{N}$ and $\operatorname{Spir}\left[\lambda, a, \theta_{0}\right] \subset h(\mathbb{D})$. By Theorem 5.7 there exists a hyperbolic petal $\Delta \subset \mathbb{D}$ such that $\operatorname{Spir}\left[\lambda, a, \theta_{0}\right] \subseteq h(\Delta)$. Moreover, by Proposition $4.16, \partial \Delta$ contains only one boundary fixed point of $\left(\phi_{t}\right)$ which is repelling. Thus, if we prove that $\sigma \in \partial \Delta$, it follows that $\sigma$ is repelling. To this aim, consider the curve $\gamma$ : $(-\infty, 0) \ni t \mapsto e^{-t \lambda+i \theta_{0}}$. Since for all $n \in \mathbb{N}$ there exists $t_{n} \in(-\infty, 0)$ such that $\gamma(t) \in$ $\operatorname{Spir}\left[\lambda, a, \theta_{0}\right] \cap\left\{w \in \mathbb{C}:|w|>R_{n}\right\} \subset V_{n}$ for all $t \leq t_{n}$, it follows that $\gamma(t)$ converges in the Carathéodory topology of $h(\mathbb{D})$ to $\hat{h}\left(\underline{x}_{\sigma}\right)$ as $t \rightarrow-\infty$. Hence, $h^{-1}(\gamma(t)) \rightarrow \sigma$ as $t \rightarrow-\infty$, proving that $\sigma \in \partial \Delta$.

As an immediate corollary of Proposition 6.1 and Theorem 6.2, we have: 
Corollary 6.3 Let $\left(\phi_{t}\right)$ be an elliptic semigroup in $\mathbb{D}$, not a group. Let $h$ be the associated Koenigs function and $\sigma \in \partial \mathbb{D}$. The following are equivalent:

(1) $\sigma$ is a super-repelling fixed point of $\left(\phi_{t}\right)$,

(2) $\lim _{z \rightarrow \sigma}|h(z)|=\infty$ and $\lim _{z \rightarrow \sigma} \operatorname{Arg}_{\lambda}(h(z))=\theta_{0}$ for some $\theta_{0} \in[-\pi, \pi]$.

Now we turn our attention to non-elliptic semigroups. The proofs of the next results are similar to those for the elliptical case, and we omit them-in fact, roughly speaking, in the non-elliptic case, the role of the modulus is played by the imaginary part and that of the $\lambda$-argument by the real part.

Theorem 6.4 Let $\left(\phi_{t}\right)$ be a non-elliptic semigroup in $\mathbb{D}$, not a group, with Denjoy-Wolff point $\tau \in \partial \mathbb{D}$ and spectral value $\lambda \geq 0$. Let $h$ be the associated Koenigs function and $\sigma \in \partial \mathbb{D}$. The following are equivalent:

(1) $\sigma$ is a repelling fixed point of $\left(\phi_{t}\right)$,

(2) $\lim _{z \rightarrow \sigma} \operatorname{Im} h(z)=-\infty$ and $-\infty<\angle \liminf _{z \rightarrow \sigma} \operatorname{Re} h(z) \neq \angle \lim \sup _{z \rightarrow \sigma} \operatorname{Re} h(z)<$ $+\infty$

(3) $\lim _{z \rightarrow \sigma} \operatorname{Im} h(z)=-\infty$ and $-\infty<\liminf _{z \rightarrow \sigma} \operatorname{Re} h(z) \neq \limsup _{z \rightarrow \sigma} \operatorname{Re} h(z)<+\infty$.

Moreover, if $\sigma$ is a repelling fixed point for $\left(\phi_{t}\right)$ with repelling spectral value $v \in(-\infty, 0)$, then there exists $a \in \mathbb{R}$ such that if $\left\{z_{n}\right\} \subset \mathbb{D}$ is a sequence converging to $\sigma$ and $\lim _{n \rightarrow \infty} \operatorname{Arg}\left(1-\bar{\sigma} z_{n}\right)=\beta \in(-\pi / 2, \pi / 2)$, then

$$
\lim _{n \rightarrow \infty} \operatorname{Re} h\left(z_{n}\right)=a+\frac{\beta}{v} \text {. }
$$

As a corollary, we have:

Corollary 6.5 Let $\left(\phi_{t}\right)$ be a non-elliptic semigroup in $\mathbb{D}$, not a group. Let $h$ be the associated Koenigs function and $\sigma \in \partial \mathbb{D}$. The following are equivalent:

(1) $\sigma$ is a super-repelling fixed point of $\left(\phi_{t}\right)$,

(2) $\lim _{z \rightarrow \sigma} \operatorname{Im} h(z)=-\infty$ and $\lim _{z \rightarrow \sigma} \operatorname{Re} h(z)=$ a for some $a \in \mathbb{R}$.

The previous results allow also to easily prove the following:

Corollary 6.6 Let $\left(\phi_{t}\right)$ be a semigroup, not an elliptic group, in $\mathbb{D}$ with Denjoy-Wolff point $\tau \in \overline{\mathbb{D}}$, and let $h$ be its Koenigs function. Let $\sigma \in \partial \mathbb{D}$. Then, $\sigma$ is not a fixed point of $\left(\phi_{t}\right)$ if and only if $\angle \lim _{z \rightarrow \sigma} h(z) \in \mathbb{C}$. Moreover, in this case,

(1) if $\tau \in \mathbb{D}$ then $\lim _{z \rightarrow \sigma} \operatorname{Arg}_{\lambda} h(z)$ exists, where $\lambda$ is the spectral value of $\left(\phi_{t}\right)$;

(2) if $\tau \in \partial \mathbb{D}$ then $\lim _{z \rightarrow \sigma} \operatorname{Re} h(z)$ exists.

\section{Examples}

Let $\left(\phi_{t}\right)$ be a semigroup, not a group, in $\mathbb{D}$, and let $h$ be its Koenigs function. Recall that, by Theorems 5.6 and 5.7, there is a one-to-one correspondence between hyperbolic petals of $\left(\phi_{t}\right)$ and maximal strips in the non-elliptic case (or maximal spirallike sectors in the elliptic case) in $h(\mathbb{D})$. Moreover, the repelling spectral value can be read by the width of the strip (or the angle of the spirallike sector). Also, by Theorem 5.8, there is a one-to-one correspondence between parabolic petals and maximal half-planes. The previous developed theory allows also to read information on the boundary of a petal using directly the image of $h$. We summarize and translate here the results in a suitable manageable way. We start with the elliptic case (recall Definition 6.1): 
Proposition 7.1 Let $\left(\phi_{t}\right)$ be an elliptic semigroup, not a group, with Denjoy-Wolff point $\tau \in \mathbb{D}$ and spectral value $\lambda \in \mathbb{C}, \operatorname{Re} \lambda>0$ and let $h$ be its Koenigs function. Let $\Delta$ be a hyperbolic petal which corresponds to the maximal spirallike sector $\operatorname{Spir}\left[\mu, 2 \alpha, \theta_{0}\right]$, for some $\alpha \in[0, \pi)$ and $\theta_{0} \in[-\pi, \pi)$. Let $\sigma \in \partial \mathbb{D} \cap \partial \Delta$ be the only repelling fixed point of $\left(\phi_{t}\right)$ contained in $\partial \Delta$. Let $\left.S:=\operatorname{spir}_{\lambda}\left[e^{i\left(\theta_{0}+\alpha\right.}\right)\right] \backslash\{0\}$ or $\left.S:=\operatorname{spir}_{\lambda}\left[e^{i\left(\theta_{0}-\alpha\right.}\right)\right] \backslash\{0\}$. Then, one and only one of the following happens:

(1) There exists $a>0$ such that $S \cap\{w \in \mathbb{C}:|w|<a\} \subset h(\mathbb{D})$ and $S \cap\{w \in \mathbb{C}:|w| \geq$ $a\} \cap h(\mathbb{D})=\emptyset$. This is the case if and only if $h^{-1}(S \cap\{w \in \mathbb{C}:|w|<a\})$ is a connected component of $\partial \Delta \cap(\mathbb{D} \backslash\{\tau\})$ whose closure is a Jordan arc with end points $\tau$ and a non-fixed point $p \in \partial \mathbb{D}$ such that $\angle \lim _{z \rightarrow p} h(z)=S \cap\{w \in \mathbb{C}:|w|=a\}$.

(2) $S \subset h(\mathbb{D})$. This is the case if and only if $h^{-1}(S)$ is a connected component of $\partial \Delta \cap(\mathbb{D} \backslash\{\tau\})$ whose closure is a Jordan arc with end points $\tau$ and $\sigma$.

Proof Assume $\left.S:=\operatorname{spir}_{\lambda}\left[e^{i\left(\theta_{0}+\alpha\right.}\right)\right] \backslash\{0\}$ (the other case is similar).

(1) Clearly, $h^{-1}(S \cap\{w \in \mathbb{C}:|w|<a\})$ is a connected component of $\partial \Delta \cap(\mathbb{D} \backslash\{\tau\})$. By Corollary 4.17, the closure of $h^{-1}(S \cap\{w \in \mathbb{C}:|w|<a\})$ is a Jordan arc joining $\tau$ with a point $p \in \partial \mathbb{D}$ which can be either $\sigma$ or a non-fixed point. Let $\gamma:\left(-\infty, t_{0}\right) \ni t \mapsto$ $e^{\lambda t+i\left(\theta_{0}+\alpha\right)}$ be a parameterization of $S$, with $t_{0} \in \mathbb{R}$ such that $e^{\lambda t_{0}+i\left(\theta_{0}+\alpha\right)}=S \cap\{w \in$ $\mathbb{C}:|w|=a\}$. Then, $\lim _{t \rightarrow t_{0}} h^{-1}(\gamma(t))=p$. Since $\lim _{t \rightarrow t_{0}} h\left(h^{-1}(\gamma(t))\right)=S \cap\{w \in$ $\mathbb{C}:|w|=a\}$, Lehto-Virtanen Theorem implies that $\angle \lim _{z \rightarrow p} h(z)=S \cap\{w \in \mathbb{C}:$ $|w|=a\}$. In particular, $\angle \lim _{z \rightarrow p}|h(z)|<+\infty$, and hence $p$ is not a fixed point by Proposition 6.1.

(2) The argument is similar and we leave the proof to the reader.

A similar argument allows to handle the non-elliptic case:

Proposition 7.2 Let $\left(\phi_{t}\right)$ be a non-elliptic semigroup, not a group, with Denjoy-Wolff point $\tau \in \partial \mathbb{D}$, and let $h$ be its Koenigs function. Let $\Delta$ be a hyperbolic petal which corresponds to the maximal strip $S=\left\{w \in \mathbb{C}: a_{1}<\operatorname{Re} w<a_{2}\right\}$ for some $a_{1}, a_{2} \in \mathbb{R}, a_{1}<a_{2}$. Let $\sigma \in \partial \mathbb{D} \cap \partial \Delta$ be the only repelling fixed point of $\left(\phi_{t}\right)$ contained in $\bar{\Delta}$. Fix $j \in\{1,2\}$. Then, one and only one of the following happens for $j=1,2$ :

(1) $\left\{w \in \mathbb{C}: \operatorname{Re} w=a_{j}\right\} \cap h(\mathbb{D})=\emptyset$.

(2) There exists $r \in \mathbb{R}$ such that $\left\{w \in \mathbb{C}: \operatorname{Re} w=a_{j}, \operatorname{Im} w>r\right\} \subset h(\mathbb{D})$ and $\{w \in \mathbb{C}$ : $\left.\operatorname{Re} w=a_{j}, \operatorname{Im} w \leq r\right\} \cap h(\mathbb{D})=\emptyset$. This is the case if and only if $h^{-1}(\{w \in \mathbb{C}: \operatorname{Re} w=$ $\left.\left.a_{j}, \operatorname{Im} w>r\right\}\right)$ is a connected component of $\partial \Delta \cap \mathbb{D}$ whose closure is a Jordan arc with end points $\tau$ and a non-fixed point $p \in \partial \mathbb{D}$ such that $\angle \lim _{z \rightarrow p} h(z)=a_{j}+i$.

(3) $\left\{w \in \mathbb{C}: \operatorname{Re} w=a_{j}\right\} \subset h(\mathbb{D})$. This is the case if and only if $h^{-1}\left(\left\{w \in \mathbb{C}: \operatorname{Re} w=a_{j}\right\}\right)$ is a connected component of $\partial \Delta \cap \mathbb{D}$ whose closure is a Jordan arc with end points $\tau$ and $\sigma$.

Finally, we have the parabolic petals:

Proposition 7.3 Let $\left(\phi_{t}\right)$ be a parabolic semigroup, not a group, with Denjoy-Wolff point $\tau \in \partial \mathbb{D}$, and let $h$ be its Koenigs function. Let $\Delta$ be a parabolic petal which corresponds to the maximal half-plane $H=\{w \in \mathbb{C}: \operatorname{Re} w>a\}$ for some $a \in \mathbb{R}$. Then, one and only one of the following happens:

(1) There exists $r \in \mathbb{R}$ such that $\{w \in \mathbb{C}: \operatorname{Re} w=a, \operatorname{Im} w>r\} \subset h(\mathbb{D})$ and $\{w \in \mathbb{C}$ : $\operatorname{Re} w=a, \operatorname{Im} w \leq r\} \cap h(\mathbb{D})=\emptyset$. This is the case if and only if $h^{-1}(\{w \in \mathbb{C}: \operatorname{Re} w=$ $a, \operatorname{Im} w>r\})$ is a connected component of $\partial \Delta \cap \mathbb{D}$ whose closure is a Jordan arc with end points $\tau$ and a non-fixed point $p \in \partial \mathbb{D}$ such that $\angle \lim _{z \rightarrow p} h(z)=a+i$. 
(2) $\{w \in \mathbb{C}: \operatorname{Re} w=a\} \subset h(\mathbb{D})$. This is the case if and only if $h^{-1}(\{w \in \mathbb{C}: \operatorname{Re} w=a\})$ is a connected component of $\partial \Delta \cap \mathbb{D}$ whose closure $J$ is a Jordan curve with $J \cap \partial \mathbb{D}=\{\tau\}$.

Proof The proof is similar to that of Proposition 7.1, so we leave it to the reader. The only issue here is to show that the case $\{w \in \mathbb{C}: \operatorname{Re} w=a\} \cap h(\mathbb{D})=\emptyset$ cannot happen. Indeed, if this is the case, then $h(\mathbb{D})=H$ and $\left(\phi_{t}\right)$ is a parabolic group.

A similar result holds in case the maximal half-plane associated with the parabolic petal is $H=\{w \in \mathbb{C}: \operatorname{Re} w<a\}$ for some $a \in \mathbb{R}$.

We give now a list of examples of petals of all types described in Proposition 4.15.

Example 7.4 Let $h$ be the Koebe function $h(z):=\frac{z}{(1-z)^{2}}, z \in \mathbb{D}$. It is univalent and $h(\mathbb{D})=\mathbb{C} \backslash(-\infty,-1 / 4]$. Consider the semigroup whose model is $\left(\mathbb{C}, h, e^{-t} z\right)$, that is, $\phi_{t}(z):=h^{-1}\left(e^{-t} h(z)\right)$, for all $z \in \mathbb{D}$ and $t \geq 0$. Since $\cap_{t \geq 0} e^{-t} h(\mathbb{D}) \backslash\{0\}=\mathbb{C} \backslash(-\infty, 0]=$ $\operatorname{Spir}[-1,-\pi, 2 \pi]$ and it is a maximal spirallike sector of $h(\mathbb{D})$, Theorem 5.7 shows that

$$
h^{-1}(\operatorname{Spir}[-1,-\pi, 2 \pi])=\mathbb{D} \backslash(-1,0]
$$

is a hyperbolic petal for $\left(\phi_{t}\right)$. Clearly, it is the unique petal of the semigroup. Therefore, $\left(\phi_{t}\right)$ has a unique boundary fixed point $\sigma \in \partial \mathbb{D}$, which is repelling with repelling spectral value $-2 \pi$. Since $\lim _{(0,1) \ni r \rightarrow 1} h(r)=\infty$, we get $\sigma=1$ by Proposition 4.16 . This petal is an example of the type described in Proposition 4.15(1).

Example 7.5 Consider the domain

$$
\Omega=\mathbb{S} \cup(1+i \mathbb{R}) \cup\{w \in \mathbb{S}+1: \operatorname{Im} w(1-\operatorname{Re} w)>1\} \subset \mathbb{S}_{2} .
$$

Let $h: \mathbb{D} \rightarrow \mathbb{C}$ be univalent such that $h(\mathbb{D})=\Omega$. By construction, $\Omega+i t \subset \Omega$ for all $t \geq 0$ and $\cup_{t \geq 0}(\Omega-i t)=\mathbb{S}_{2}$. Consider the semigroup whose model is $\left(\mathbb{S}_{2}, h, z+i t\right)$, that is, $\phi_{t}(z):=h^{-1}(h(z)+i t)$, for all $z \in \mathbb{D}$ and $t \geq 0$. Let us call $\tau \in \partial \mathbb{D}$ its Denjoy-Wolff point. Since $\cap_{t \geq 0}(\Omega+i t)=\cup_{x \in(0,1]}(x+i \mathbb{R})=\mathbb{S} \cup(1+i \mathbb{R}), h(\mathbb{D})$ contains a unique maximal strip, $\mathbb{S}$, whose boundary is $i \mathbb{R} \cup(1+i \mathbb{R})$. Let $\Delta=h^{-1}(\mathbb{S})$. Therefore, $\Delta$ is the unique hyperbolic petal of the semigroup $\left(\phi_{t}\right)$. Let us denote by $\sigma$ the repelling fixed point associated with $\Delta$ given by Proposition 4.16. By Proposition 7.2, the maximal invariant curve $\mathbb{R} \ni t \mapsto h^{-1}(1+i t)$ is a connected component of $\partial \Delta \cap \mathbb{D}$ whose closure is a Jordan arc with end points $\tau$ and $\sigma$. It divides the unit disc in two connected components, one of them is the petal, and the other one is $B=h^{-1}(\{w \in \mathbb{S}+1: \operatorname{Im} w(1-\operatorname{Re} w)>1\})$. Clearly, $\bar{\Delta} \cap \partial \mathbb{D}$ and $\bar{B} \cap \partial \mathbb{D}$ are the two Jordan arcs in $\partial \mathbb{D}$ that joins $\sigma$ and $\tau$. Let us denote by $J$ the one which is included in $\partial \Delta$. Then, $\partial \Delta=\{\tau, \sigma\} \cup h^{-1}(1+i \mathbb{R}) \cup J$. Thus, $\Delta$ is an example of a petal of the type described in Proposition 4.15(2).

Example 7.6 Consider the domain

$$
\Omega=\{w \in \mathbb{S}: \operatorname{Im} w>0\} \cup(1+i(0,+\infty)) \cup(\mathbb{H}+1) \subset \mathbb{H} .
$$

Let $h: \mathbb{D} \rightarrow \mathbb{C}$ be univalent such that $h(\mathbb{D})=\Omega$. Since $\bar{\Omega}^{\infty}$ is a Jordan domain, there exists a homeomorphism $\tilde{h}: \overline{\mathbb{D}} \rightarrow \bar{\Omega}^{\infty}$ such that $\left.\tilde{h}\right|_{\mathbb{D}}=h$. Let $\left(\phi_{t}\right)$ be the parabolic semigroup whose model is $(\mathbb{H}, h, z+i t)$, that is, $\phi_{t}(z):=h^{-1}(h(z)+i t)$, for all $z \in \mathbb{D}$ and $t \geq 0$. Let $\tau \in \partial \mathbb{D}$ be its Denjoy-Wolff point. Note that $\cap_{t \geq 0}(\Omega+i t)=\cup_{x \in(1,+\infty)}(x+i \mathbb{R})=\mathbb{H}+1$. Let $\Delta=h^{-1}(\mathbb{H}+1)$. Therefore, $\Delta$ is the unique (parabolic) petal of the semigroup $\left(\phi_{t}\right)$. Write $J=h^{-1}(1+i(0,+\infty)) \subset \mathbb{D}, \sigma=\tilde{h}^{-1}(1)$ and $A=\tilde{h}^{-1}(1+i(-\infty, 0)) \subset \partial \mathbb{D}$. Then, $\partial \Delta=\{\tau\} \cup J \cup\{\sigma\} \cup A$. Thus, $\Delta$ is an example of a petal described in Proposition 4.15(2). The difference with Example 7.5 is that $\sigma$ in this case is not a boundary fixed point. 
Example 7.7 Consider the domain

$$
\begin{aligned}
\Omega=\{w & \in \mathbb{S}: \operatorname{Im} w(\operatorname{Re} w-1)<\operatorname{Re} w\} \cup(1+i \mathbb{R}) \cup(\mathbb{S}+1) \cup \\
& \cup(2+i \mathbb{R}) \cup\{w \in \mathbb{S}+2: \operatorname{Im} w(\operatorname{Re} w-2)>\operatorname{Re} w-3\} \subset \mathbb{S}_{3} .
\end{aligned}
$$

Let $h: \mathbb{D} \rightarrow \mathbb{C}$ be univalent such that $h(\mathbb{D})=\Omega$. By construction, $\Omega+i t \subset \Omega$ for all $t \geq 0$ and $\cup_{t \geq 0}(\Omega-i t)=\mathbb{S}_{3}$. Consider the semigroup whose model is $\left(\mathbb{S}_{3}, h, z+i t\right)$, that is, $\phi_{t}(z):=h^{-1}(h(z)+i t)$, for all $z \in \mathbb{D}$ and $t \geq 0$. Let $\tau \in \partial \mathbb{D}$ be its Denjoy-Wolff point. Since $\cap_{t \geq 0}(\Omega+i t)=\cup_{x \in[1,2]}(x+i \mathbb{R})$, its interior has a unique maximal strip whose boundary is $(1+i \mathbb{R}) \cup(2+i \mathbb{R})$. Let $\Delta=h^{-1}\left(\cup_{x \in(1,2)}(x+i \mathbb{R})\right)=h^{-1}(\mathbb{S}+1)$. Therefore, $\Delta$ is the unique (hyperbolic) petal of the semigroup $\left(\phi_{t}\right)$. Let us denote by $\sigma$ the repelling fixed point associated with $\Delta$ given by Proposition 4.16. By Proposition 7.2(3), $h^{-1}(1+i \mathbb{R})$ and $h^{-1}(2+i \mathbb{R})$ are connected components of $\partial \Delta \cap \mathbb{D}$ whose closures are Jordan arcs with end points $\tau$ and $\sigma$. According to Proposition 4.15, $\partial \Delta=\{\tau, \sigma\} \cup h^{-1}(1+i \mathbb{R}) \cup h^{-1}(2+i \mathbb{R})$. Thus, $\Delta$ is an example of a petal described in Proposition 4.15(3).

Example 7.8 Consider the domain

$$
\begin{array}{r}
\Omega=\{w \in \mathbb{S}: \operatorname{Im} w>0\} \cup(1+i(0,+\infty)) \cup(\mathbb{S}+1) \cup \\
\cup(2+i(0,+\infty)) \cup\{w \in \mathbb{S}+2: \operatorname{Im} w>0\} \subset \mathbb{S}_{3} .
\end{array}
$$

Let $h: \mathbb{D} \rightarrow \mathbb{C}$ be univalent such that $h(\mathbb{D})=\Omega$. Consider the semigroup whose model is $\left(\mathbb{S}_{3}, h, z+i t\right)$, that is, $\phi_{t}(z):=h^{-1}(h(z)+i t)$, for all $z \in \mathbb{D}$ and $t \geq 0$. Denote by $\tau \in \partial \mathbb{D}$ its Denjoy-Wolff point. Note that $\cap_{t \geq 0}(\Omega+i t)=\cup_{x \in(1,2)}(x+i \mathbb{R})=\mathbb{S}+1$. Let $\Delta=h^{-1}(\mathbb{S}+1)$. Therefore, $\Delta$ is the unique hyperbolic petal of the semigroup $\left(\phi_{t}\right)$. Let us denote by $\sigma$ the repelling fixed point associated with $\Delta$ given by Proposition 4.16. Write $J_{j}=h^{-1}(j+i(0,+\infty)) \subset \mathbb{D}, j=1,2$. Proposition 7.2(2) guarantees that $J_{1}$ and $J_{2}$ are connected components of $\partial \Delta \cap \mathbb{D}$ with end points $\tau$ and a non-fixed point $p_{j} \in \partial \mathbb{D}$ such that $\angle \lim _{z \rightarrow p_{j}} h(z)=j+i r$. By default, looking at Proposition 4.15, $\Delta$ is an example of a described in statement (4). Therefore, if $A \subset \partial \mathbb{D}$ is the closed arc with end points $p_{1}$ and $p_{2}$ which does not pass through $\tau$, we have that $\partial \Delta=\{\tau\} \cup J_{1} \cup J_{2} \cup A$.

Example 7.9 Consider the domain

$$
\Omega=\{w \in \mathbb{S}: \operatorname{Im} w(\operatorname{Re} w-1)<\operatorname{Re} w\} \cup \overline{(\mathbb{H}+1)} \subset \mathbb{H} .
$$

Let $h: \mathbb{D} \rightarrow \mathbb{C}$ be univalent such that $h(\mathbb{D})=\Omega$. Consider the parabolic semigroup whose model is $(\mathbb{H}, h, z+i t)$, that is, $\phi_{t}(z):=h^{-1}(h(z)+i t)$, for all $z \in \mathbb{D}$ and $t \geq 0$. Let $\tau \in \partial \mathbb{D}$ be its Denjoy-Wolff point. Note that $\cap_{t \geq 0}(\Omega+i t)=\cup_{x \in[1,+\infty)}(x+i \mathbb{R})=\overline{\mathbb{H}}+1$ and write $\Delta=h^{-1}(\mathbb{H}+1)$. Therefore, $\Delta$ is the unique parabolic petal of the semigroup $\left(\phi_{t}\right)$. Let $A=h^{-1}(1+i \mathbb{R}) \subset \mathbb{D}$. Then, Proposition 7.3(2) shows that $A$ is a connected component of $\partial \Delta \cap \mathbb{D}$ and $\partial \Delta=\{\tau\} \cup A$. Thus, $\Delta$ is an example of a petal described in Proposition $4.15(5)$.

\section{An example of a semigroup with a non-regular pre-model}

We end up this paper by constructing an example of a semigroup with a repelling fixed point $\sigma$, a pre-model $\left(\mathbb{D}, g, \eta_{t}\right)$ for $\left(\phi_{t}\right)$ at $\sigma$ such that $g$ is not regular at $\sigma$.

Some remarks are in order. Let $\left(\mathbb{D}, g, \eta_{t}\right)$ be a pre-model for $\left(\phi_{t}\right)$ at a repelling fixed point $\sigma \in \partial \mathbb{D}$. Let $\Delta=g(\mathbb{D}) \subset \mathbb{D}$ be the associated petal. 
Since by (2) in the definition of pre-model $\angle \lim _{z \rightarrow \sigma} g(z)=\sigma$, by definition, $g$ is regular at $\sigma$ if $\angle \lim _{z \rightarrow \sigma} \frac{\sigma-g(z)}{\sigma-z}$ exists (finite). By the Julia-Wolff-Carathéodory theorem, this condition is equivalent to $\alpha:=\liminf _{z \rightarrow \sigma} \frac{1-|g(z)|}{1-|z|}<+\infty$ and, in fact, $\angle \lim _{z \rightarrow \sigma} \frac{\sigma-g(z)}{\sigma-z}=\alpha$. Since $\eta_{-t}(z)$ converges to $\sigma$ non-tangentially as $t \rightarrow+\infty$ for every $z \in \mathbb{D}$, the previous argument implies that $g$ is regular at $\sigma$ if and only if $\lim \sup _{t \rightarrow+\infty} \frac{1-\left|g\left(\eta_{-t}(z)\right)\right|}{1-\left|\eta_{-t}(z)\right|}<+\infty$ for every $z \in \mathbb{D}$, which, in turn, is equivalent to

$$
\limsup _{t \rightarrow+\infty}\left[\omega\left(0, \eta_{-t}(z)\right)-\omega\left(0, g\left(\eta_{-t}(z)\right)\right)\right]<+\infty, \quad \forall z \in \mathbb{D} .
$$

The map $g$ is a biholomorphism on the image-hence an isometry for the hyperbolic distance-and $g \circ \eta_{-t}=\left(\left.\phi_{t}\right|_{\Delta}\right)^{-1} \circ g$ for all $t \geq 0$. Therefore,

$$
\omega\left(0, \eta_{-t}(z)\right)=k_{\Delta}\left(g(0), g\left(\eta_{-t}(z)\right)\right)=k_{\Delta}\left(g(0),\left(\left.\phi_{t}\right|_{\Delta}\right)^{-1}(g(z))\right) .
$$

If the semigroup $\left(\phi_{t}\right)$ is parabolic, with universal model ( $\left.\mathbb{C}, h, z+i t\right)$, then by Theorem 5.6(2), $h(\Delta)$ is a maximal strip $S \subset h(\mathbb{D})$. Therefore, taking into account that $h \circ\left(\left.\phi_{t}\right|_{\Delta}\right)^{-1}(g(z))=$ $h(g(z))-i$ for all $t \geq 0$, it turns out that (8.1) —and $g$ being regular at $\sigma$-is equivalent to

$$
\limsup _{t \rightarrow+\infty}\left[k_{S}(h(g(0)), h(g(z))-i t)-k_{h(\mathbb{D})}(h(0), h(g(z))-i t)\right]<+\infty, \quad \forall z \in \mathbb{D},
$$

and, using the triangle inequality, setting $w:=h(g(z))$, this latter inequality is equivalent to

$$
\limsup _{t \rightarrow+\infty}\left[k_{S}(w, w-i t)-k_{h(\mathbb{D})}(w, w-i t)\right]<+\infty \quad \forall w \in S .
$$

We construct our example as follows. Let $\left\{y_{k}\right\}$ be a strictly decreasing sequence of real numbers, to be suitable chosen later, such that $\lim _{k \rightarrow \infty} y_{k}=-\infty$. For $k=1,2, \ldots$, let

$$
D_{k}:=\mathbb{C} \backslash\left\{z \in \mathbb{C}: \operatorname{Re} z= \pm\left(1+\frac{1}{k}\right), \operatorname{Im} z \leq y_{k}\right\}
$$

Let $D:=\bigcap_{k} D_{k}$. Note that $D$ is a simply connected domain, $D+i t \subset D$ for all $t \geq 0$, and $D$ is symmetric with respect to the imaginary axis. Moreover, $D$ contains a maximal strip $S:=\{z \in \mathbb{C}:-1<\operatorname{Re} z<1\}$.

Let $h: \mathbb{D} \rightarrow D$ be a Riemann map. Setting $\left.\phi_{t}(z):=h^{-1}(h(z)+i t)\right), z \in \mathbb{D}, t \geq 0$, it follows that $\left(\phi_{t}\right)$ is a holomorphic semigroup of $\mathbb{D}$ with universal model $(\mathbb{C}, h, z+i t)$. By Theorem 5.7, the maximal strip $S$ in $D$ corresponds to a hyperbolic petal, whose closure contains a unique repelling point of $\left(\phi_{t}\right)$, say $\sigma \in \partial \mathbb{D}$. Let $\left(\mathbb{D}, g, \eta_{t}\right)$ be a pre-model for $\left(\phi_{t}\right)$ at $\sigma$. According to (8.2), $g$ is not regular at $\sigma$ if

$$
\limsup _{t \rightarrow+\infty}\left[k_{S}(0,-i t)-k_{D}(0,-i t)\right]=+\infty .
$$

Recall that, if $r>0$ and $S_{2 r}=\{w \in \mathbb{C}:-r<\operatorname{Re} w<r\}$, then for all $s, t \in \mathbb{R}$,

$$
k_{S_{2 r}}(i s, i t)=\frac{\pi}{4 r}|t-s| .
$$

Let $\Omega$ be a simply connected domain in $\mathbb{C}, \Omega \neq \mathbb{C}$. Given $p \in \Omega$ and $R>0$, we denote by $D^{\text {hyp }}(p, R)$ the hyperbolic disc of center $p$ and radius $R$, that is, $D^{\text {hyp }}(p, R):=\{z \in$ $\left.\Omega: k_{\Omega}(z, p)<R\right\}$. Since $\cup_{R>0} D^{h y p}(p, R)=\Omega$, it follows that for every $z, w \in \Omega$, $\lim _{R \rightarrow+\infty} k_{D^{h y p}(p, R)}(z, w)=k_{\Omega}(z, w)$. From this observation, it is not hard to prove the following lemma: 
Lemma 8.1 For all $c>1$ and $M>0$, there exists $R>0$ such that for all $p \in \Omega$,

$$
k_{D^{h y p}(p, R)}(z, w) \leq c k_{\Omega}(z, w)
$$

for all $z, w \in D^{\text {hyp }}(p, M)$.

Now we need a localization lemma which allows to suitably choose the sequence $\left\{y_{n}\right\}$ :

Lemma 8.2 There exist two strictly decreasing sequences $\left\{y_{k}\right\}$ and $\left\{\alpha_{k}\right\}$ of real numbers, both converging to $-\infty$, such that, for each $k \in \mathbb{N}$,

$$
k_{D}\left(\left(\alpha_{k}+\frac{1}{2}\right) i,\left(\alpha_{k}-\frac{1}{2}\right) i\right) \leq\left(1+\frac{1}{2 k}\right) k_{D_{k}}\left(\left(\alpha_{k}+\frac{1}{2}\right) i,\left(\alpha_{k}-\frac{1}{2}\right) i\right),
$$

where $D_{k}$ is defined by (8.3).

Proof Given $p \in D_{k}$ and $R>0$, let $B_{k}(p, R):=\left\{w \in D_{k}: k_{D_{k}}(p, w)<R\right\}$. Let $S_{k}:=\{w \in \mathbb{C}:|\operatorname{Re} z|<1+1 / k\}$. Clearly $S_{k} \subset \Omega_{k}$ for all $k$. Hence, for every $\alpha \in \mathbb{R}$,

$$
k_{D_{k}}((\alpha+1 / 2) i, \alpha i) \leq k_{S_{k}}((\alpha+1 / 2) i, \alpha i)=\frac{\pi}{8(1+1 / k)}<\pi
$$

Set $y_{1}=0$ and $\alpha_{1}=-1 / 2$. By Lemma 8.1 applied to $D_{1}$ and the invariance of hyperbolic distance with respect to biholomorphisms, there exists $R_{1}>0$ such that for all $z, w \in$ $B_{1}(-i / 2, \pi)$, we have $k_{B_{1}\left(-i / 2, R_{1}\right)}(z, w) \leq\left(1+\frac{1}{2}\right) k_{D_{1}}(z, w)$. In particular, by (8.5),

$$
k_{B_{1}\left(-i / 2, R_{1}\right)}(0,-i) \leq\left(1+\frac{1}{2}\right) k_{D_{1}}(0,-i) .
$$

Since the closure of $B_{1}\left(-i / 2, R_{1}\right)$ is compact in $D_{1}, \beta_{1}:=\inf \left\{\operatorname{Im} w: w \in B_{1}\left(-i / 2, R_{1}\right)\right\}>$ $-\infty$.

If $y_{j}<\beta_{1}$ for all $j \geq 2$, then $B_{1}\left(-i / 2, R_{1}\right) \subset D$. Hence, $k_{D}(0,-i) \leq$ $k_{B_{1}\left(-i / 2, R_{1}\right)}(0,-i)$ and the statement holds for $k=1$ by (8.6).

Now we choose $y_{2}$ and $\alpha_{2}$. Let $y_{2}<\beta_{1}$ and let $f_{2}: \mathbb{D} \rightarrow D_{2}$ be a Riemann map. By [26, Lemma 2, p. 162], there exists $p:=\lim _{t \rightarrow-\infty} f_{2}^{-1}(i t)$. Let $\Gamma:=f_{2}^{-1}\left(D_{2} \backslash D_{1}\right)$.

For each $n=0,1, \ldots$, let $J_{n}$ be the segment in $D_{2}$ with extreme points $y_{2} \pm(1+1 / 2-n) i$ and $C_{n}=f_{2}^{-1}\left(J_{n}\right)$. Then, $\left(C_{n}\right)$ is a null chain in $\mathbb{D}$ and its impression is the singleton $p$. Clearly, $\bar{\Gamma}$ does not intersect the interior part of $C_{n}$ for all $n$ and then $p \notin \bar{\Gamma}$.

Let $R_{2}$ be given by Lemma 8.1 applied to $\mathbb{D}$ with $M=\pi$ and $c=1+1 / 4$. Since $p \notin \bar{\Gamma}$, there exists $\alpha_{2}<y_{2}$ such that $D^{h y p}\left(f_{2}^{-1}\left(\alpha_{2} i\right), R_{2}\right) \cap \bar{\Gamma}=\emptyset$. Hence, $B_{2}\left(\alpha_{2} i, R_{2}\right) \subset D_{1} \cap D_{2}$. Therefore, arguing as before, the statement holds for $k=2$.

Let $\beta_{2}=\inf \left\{\operatorname{Im} w: w \in B_{2}\left(\alpha_{2} i, R_{2}\right)\right\}>-\infty$. Since $\alpha_{2}<y_{2}<\beta_{1}$, we get that $\beta_{2}<\beta_{1}$ and the lemma is proved repeating the previous argument by induction.

Now, choose the sequences $\left\{y_{k}\right\},\left\{\alpha_{k}\right\}$ as in the previous lemma. We may (and do) assume that $\alpha_{k+1}<\alpha_{k}-1$ for all $k$. Taking into account that $D$ and $S$ are symmetric with respect to the imaginary axis, hence the imaginary axis is a geodesic for the hyperbolic distance, we have 


$$
\begin{aligned}
& k_{S}\left(0, i\left(\alpha_{k}+1 / 2\right)\right)-k_{D}\left(0, i\left(\alpha_{k}+1 / 2\right)\right) \\
&=\sum_{j=1}^{k-1}\left[k_{S}\left(i\left(\alpha_{j}-1 / 2\right), i\left(\alpha_{j}+1 / 2\right)\right)+k_{S}\left(i\left(\alpha_{j}-1 / 2\right), i\left(\alpha_{j+1}+1 / 2\right)\right)\right. \\
&\left.\quad-k_{D}\left(i\left(\alpha_{j}-1 / 2\right), i\left(\alpha_{j}+1 / 2\right)\right)-k_{D}\left(i\left(\alpha_{j}-1 / 2\right), i\left(\alpha_{j+1}+1 / 2\right)\right)\right] \\
& \geq \sum_{j=1}^{k-1}\left[k_{S}\left(i\left(\alpha_{j}-1 / 2\right), i\left(\alpha_{j}+1 / 2\right)\right)-k_{D}\left(i\left(\alpha_{j}-1 / 2\right), i\left(\alpha_{j}+1 / 2\right)\right)\right] \\
& \geq \sum_{j=1}^{k-1}\left[k_{S}\left(i\left(\alpha_{j}-1 / 2\right), i\left(\alpha_{j}+1 / 2\right)\right)-\left(1+\frac{1}{2 j}\right) k_{D_{j}}\left(i\left(\alpha_{j}-1 / 2\right), i\left(\alpha_{j}+1 / 2\right)\right)\right] \\
& \geq \sum_{j=1}^{k-1}\left[k_{S}\left(i\left(\alpha_{j}-1 / 2\right), i\left(\alpha_{j}+1 / 2\right)\right)-\left(1+\frac{1}{2 j}\right) k_{S_{j}}\left(i\left(\alpha_{j}-1 / 2\right), i\left(\alpha_{j}+1 / 2\right)\right)\right] \\
&= \sum_{j=1}^{k-1}\left[\frac{\pi}{4}-\left(1+\frac{1}{2 j}\right) \frac{\pi}{4\left(1+\frac{1}{j}\right)}\right]=\frac{\pi}{8} \sum_{j=1}^{k-1} \frac{1}{1+j},
\end{aligned}
$$

and (8.4) holds.

Therefore, $g$ is not regular at $\sigma$. Note that, by Remark 4.19, for every other pre-model $\left(\mathbb{D}, \tilde{g}, \eta_{t}\right)$ for $\left(\phi_{t}\right)$ at $\sigma$ the map $\tilde{g}$ is not regular at $\sigma$.

\section{References}

1. Abate, M.: Iteration Theory of Holomorphic Maps on Taut Manifolds. Mediterranean Press, Rende (1989)

2. Arosio, L.: Canonical models for the forward and backward iteration of holomorphic maps. J. Geom. Anal. 27(2), 1178-1210 (2017)

3. Arosio, L., Bracci, F.: Canonical models for holomorphic iteration. Trans. Am. Math. Soc. 5(368), 33053339 (2016)

4. Berkson, E., Porta, H.: Semigroups of holomorphic functions and composition operators. Mich. Math. J. 25, 101-115 (1978)

5. Bracci, F.: Fixed points of commuting holomorphic mappings other than the Wolff point. Trans. Am. Math. Soc. 355(6), 2569-2584 (2003)

6. Bracci, F., Gumenyuk, P.: Contact points and fractional singularities for semigroups of holomorphic self-maps in the unit disc. J. Anal. Math. 130(1), 185-217 (2016)

7. Bracci, F., Contreras, M.D., Díaz-Madrigal, S.: Topological invariants for semigroups of holomorphic self-maps of the unit disc. J. Math. Pures Appl. 107(1), 78-99 (2017)

8. Collingwood, E.F., Lohwater, A.J.: The Theory of Cluster Sets, Cambridge Tracts in Mathematics and Mathematical Physics, No. 56. Cambridge University Press, Cambridge (1966)

9. Contreras, M.D., Díaz-Madrigal, S.: Analytic flows on the unit disk: angular derivatives and boundary fixed points. Pac. J. Math. 222, 253-286 (2005)

10. Contreras, M.D., Díaz-Madrigal, S., Pommerenke, Ch.: Fixed points and boundary behavior of the Koenigs function. Ann. Acad. Sci. Fenn. Math. 29, 471-488 (2004)

11. Contreras, M.D., Díaz-Madrigal, S., Pommerenke, Ch.: On boundary critical points for semigroups of analytic functions. Math. Scand. 98, 125-142 (2006)

12. Cowen, C.C.: Iteration and the solution of functional equations for functions analytic in the unit disk. Trans. Am. Math. Soc. 265, 69-95 (1981)

13. Cowen, C.C.: Commuting analytic functions. Trans. Am. Math. Soc. 283, 685-695 (1984)

14. Cowen, C.C., MacCluer, B.D.: Composition Operators on Spaces of Analytic Functions. Studies in Advanced Mathematics. CRC Press, Boca Raton (1995)

15. Elin, M., Shoikhet, D.: Linearization Models for Complex Dynamical Systems. Topics in univalent functions, functional equations and semigroup theory. Birkhäuser, Basel (2010) 
16. Elin, M., Shoikhet, D., Zalcman, L.: A flower structure of backward flow invariant domains for semigroups. C. R. Math. Acad. Sci. Paris 346(5-6), 293-296 (2008)

17. Elin, M., Shoikhet, D., Zalcman, L.: A flower structure of backward flow invariant domains for semigroups. Ann. Acad. Sci. Fenn. Math. 33, 3-34 (2008)

18. Gumenyuk, P.: Angular and unrestricted limits of one-parameter semigroups in the unit disk. J. Math. Anal. Appl. 417, 200-224 (2014)

19. Heins, M.H.: A generalization of the Aumann-Carathodory "Starrheitssatz". Duke Math. 8, 312-316 (1941)

20. Poggi-Corradini, P.: Angular derivatives at boundary fixed points for self-maps of the disk. Proc. Am. Math. Soc. 126, 1697-1708 (1998)

21. Poggi-Corradini, P.: Canonical conjugation at fixed points other than the Denjoy-Wolff point. Ann. Acad. Sci. Fenn. Math. 25(2), 487-499 (2000)

22. Poggi-Corradini, P.: Backward-iteration sequences with bounded hyperbolic steps for analytic self-maps of the disk. Rev. Mat. Iberoam. 19(3), 943-970 (2003)

23. Poggi-Corradini, P.: Iteration of analytic self-maps of the disk: an overview. Cubo 6(1), 73-80 (2004)

24. Pommerenke, Ch.: Univalent Functions. Vandenhoeck \& Ruprecht, Göttingen (1975)

25. Pommerenke, Ch.: Boundary Behaviour of Conformal Mappings. Springer, Berlin (1992)

26. Shapiro, J.H.: Composition Operators and Classical Function Theory. Springer, New York (1993)

27. Shoikhet, D.: Semigroups in Geometrical Function Theory. Kluwer Academic Publishers, Dordrecht (2001)

28. Siskakis, A.G.: Semigroups of composition operators and the Cesàro operator on $H^{p}(D)$. Ph. D. Thesis, University of Illinois (1985)

29. Siskakis, A.G.: Semigroups of composition operators on spaces of analytic functions, a review. Contemp. Math. 213, 229-252 (1998)

\section{Affiliations}

\section{Filippo Bracci $^{1}$ (D) Manuel D. Contreras ${ }^{2}$. Santiago Díaz-Madrigal ${ }^{2}$. Hervé Gaussier ${ }^{3}$}

Manuel D. Contreras

contreras@us.es

Santiago Díaz-Madrigal

madrigal@us.es

Hervé Gaussier

herve.gaussier@univ-grenoble-alpes.fr

1 Dipartimento di Matematica, Università di Roma “Tor Vergata”, Via della Ricerca Scientifica 1, 00133 Rome, Italy

2 Camino de los Descubrimientos, s/n Departamento de Matemática Aplicada II and IMUS, Universidad de Sevilla, 41092 Seville, Spain

3 CNRS, IF, Univ. Grenoble Alpes, 38000 Grenoble, France 